\title{
Brown dwarfs as ideal candidates for detecting UV aurora outside the Solar System: Hubble Space Telescope observations of 2 MASS J1237+6526
}

\author{
Joachim Saur ${ }^{1} \odot$, Clarissa Willmes ${ }^{1}$, Christian Fischer $^{1}$, Alexandre Wennmacher ${ }^{1} \odot$, Lorenz $_{\text {Roth }}{ }^{2} \odot$, \\ Allison Youngblood ${ }^{3}$, Darrell F. Strobel ${ }^{4} \odot$, and Ansgar Reiners ${ }^{5}{ }^{\oplus}$ \\ ${ }^{1}$ Institut für Geophysik und Meteorologie, Universität zu Köln, Cologne, Germany \\ e-mail: saur@geo.uni-koeln.de \\ ${ }^{2}$ KTH Royal Institute of Technology, Stockholm, Sweden \\ ${ }^{3}$ Laboratory for Atmospheric and Space Physics, University of Colorado, Boulder, USA \\ ${ }^{4}$ Department of Earth and Planetary Sciences; Department of Physics and Astronomy, Johns Hopkins University, Baltimore, USA \\ ${ }^{5}$ Institut für Astrophysik, Georg-August-Universität, Göttingen, Germany
}

Received 23 December 2020 / Accepted 19 July 2021

\begin{abstract}
Context. Observations of auroral emissions are powerful means to remotely sense the space plasma environment around planetary bodies and ultracool dwarfs. Therefore successful searches and characterization of aurorae outside the Solar System will open new avenues in the area of extrasolar space physics.

Aims. We aim to demonstrate that brown dwarfs are ideal objects to search for UV aurora outside the Solar System. We specifically search for UV aurora on the late-type T6.5 brown dwarf 2MASS J12373919+6526148 (in the following 2MASS J1237+6526).

Methods. Introducing a parameter referred to as auroral power potential, we derive scaling models for auroral powers for rotationally driven aurora applicable to a broad range of wavelengths. We also analyze Hubble Space Telescope observations obtained with the STIS camera at near-UV, far-UV, and Ly- $\alpha$ wavelengths of 2MASS J1237+6526.

Results. We show that brown dwarfs, due to their typically strong surface magnetic fields and fast rotation, can produce auroral UV powers on the order of $10^{19}$ watt or more. Considering their negligible thermal UV emission, their potentially powerful auroral emissions make brown dwarfs ideal candidates for detecting extrasolar aurorae. We find possible emission from 2MASS J1237+6526, but cannot conclusively attribute it to the brown dwarf due to low signal-to-noise values in combination with nonsystematic trends in the background fluxes. The observations provide upper limits for the emission at various UV wavelength bands. The upper limits for the emission correspond to a UV luminosity of $\sim 1 \times 10^{19}$ watt, which lies in the range of the theoretically expected values.

Conclusions. The possible auroral emission from the dwarf could be produced by a close-in companion and/or magnetospheric transport processes.
\end{abstract}

Key words. brown dwarfs - planets and satellites: aurorae - ultraviolet: stars - ultraviolet: planetary systems stars: individual: 2MASS J12373919+652614

\section{Introduction}

Brown dwarfs are objects in the mass range between low mass stars and planets (13-80 $M_{J}$ Jupiter masses; see, e.g., Kumar 1962; Hayashi \& Nakano 1963). They possess very strong magnetic fields and are often fast rotators, which is expected to lead to large rotationally dominated magnetospheres (Pineda et al. 2017). However, very little is known about the plasma properties within the dwarfs' magnetospheres and the physical processes that energize the plasma. In the Solar System strong constraints on the planets' magnetospheres stem from remote sensing of their auroral emissions through the full electromagnetic wavelengths range. By auroral emission we refer to nonthermal emission caused by electron or ion impact excitation of atmospheric neutrals where the electrons and ions originate external to the bodies. The upper atmosphere of a planet in which the auroral emission is excited acts as a screen, and thus makes magnetospheric processes visible on the central body.
Aurorae outside of the Solar System have not been detected with certainty. H- $\alpha$ and radio emission from the ultracool dwarf 2MASS J18353790+3259545 (Lépine et al. 2003; Reid et al. 2003; Berger 2006; Berger et al. 2008), here referred to as LSR $\mathrm{J} 1835+3259$, was interpreted by Hallinan et al. (2015) as auroral emission. However, subsequent observations with the Hubble Space Telescope (HST) to confirm the ultraviolet (UV) counterpart to the expected auroral emission revealed a UV spectrum that does not resemble the auroral emission known from Jupiter, the brightest auroral emitter in the Solar System, but instead appears similar to the emission of a late-type cool star (Saur et al. 2018a). No other independent confirmation of auroral emission from LSR J1835+3259 as proposed by Hallinan et al. (2015) exists. Kao et al. $(2016,2018)$ detected circularly polarized radio emission from a number of T- and L-dwarfs. In analogy to circularly polarized radio emission caused by auroral electron beams in the Solar System, these authors attribute the radio emission from the dwarfs to auroral electric current systems. However, further confirmation is needed of whether electron or ion beams 
stemming from magnetosphere-ionosphere coupling processes in the brown dwarfs' magnetospheres are indeed the cause of the circularly polarized radio emission or if the emission is chromospheric.

We obtained observations with the HST/STIS camera at near-UV (NUV), far-UV (FUV), and Ly- $\alpha$ wavelengths of the brown dwarf 2MASS J12373919+6526148 (Burgasser et al. 1999), here referred to as 2 MASS J1237+6526. For the interpretation of these observations in the context of UV aurora outside the Solar System, we first need to develop a theoretical framework and context before the presentation of the data and its analysis. Therefore, we derive auroral luminosity estimates and demonstrate that brown dwarfs are ideal candidates to search for ultraviolet aurora outside of the Solar System. On the one hand, they possess strong kilogauss magnetic fields and are fast rotators with periods of only a few hours. We show that these conditions favor strong auroral emission. On the other hand, brown dwarfs are not massive enough to burn hydrogen as stars do and only have weak internal energy sources from fusion of deuterium and lithium. Therefore, they are too cold to generate competing thermal UV emission (for a detailed discussion of brown dwarf properties, see, e.g., Chabrier \& Baraffe 2000; Burrows et al. 2001; Kirkpatrick 2005; Reid 2013).

Expressions for radio (or auroral) emission from planetary bodies and dwarfs have been derived or applied by a number of authors. For example, Zarka et al. (2001) and Zarka (2007) developed a radio-magnetic Bode's law for planets and exoplanets. The focus of these studies is on emission driven by the flow of magnetized plasma past the bodies but not on rotationally driven plasma transport within the objects' magnetospheres. A series of authors estimated auroral powers generated by a companion for individual cases (e.g., Zarka 2007; Saur et al. 2013; Hallinan et al. 2015; Turnpenney et al. 2018; Fischer \& Saur 2019; Vedantham et al. 2020a,b) and for radio emission from ultracool dwarfs due to their fast rotation (e.g., Nichols et al. 2012; Turnpenney et al. 2017).

The brown dwarf 2MASS J1237+6526, for which we present HST observations, is a dwarf of spectral type T6.5 in the constellation Draco. It was discovered by Burgasser et al. (1999) and is located at a distance of $10.42 \pm 0.52 \mathrm{pc}$ (Vrba et al. 2004). 2MASS J1237+6526 possesses an effective temperature $T_{\text {eff }}$ of $\sim 830_{-27}^{+31} \mathrm{~K}$ (Kao et al. 2016). Its mass was estimated to be $41 \pm$ $26 M_{J}^{27}$ and its age is considered to be older than 3.4 Gyrs (Filippazzo et al. 2015; Kao et al. 2016). Burgasser et al. (2000) discovered anomalously hyperactive $\mathrm{H}-\alpha$ emission at $\log \left(L_{\mathrm{H}-\alpha} / L_{\mathrm{bol}}\right)=-4.3$ from 2MASS J1237+6526. Such intensive emission is typically seen in early M-dwarf stars, but is exceptional for late-type brown dwarfs (Gizis et al. 2000; Schmidt et al. 2015; Pineda et al. 2017). The origin of this exceptionally bright emission is still unclear. Subsequent observations of 2 MASS J1237+6526 confirmed that the H- $\alpha$ emission is persistent over at least $1.6 \mathrm{yr}$ with only mild variations within a factor of two, ruling out flaring as a cause (Burgasser et al. 2002). Liebert \& Burgasser (2007) also discarded a youthful chromospheric activity or a massive, possibly binary, companion as the origin of the H- $\alpha$ emission. Search for variability within the $J$ band rendered conflicting results (Burgasser et al. 2002; Artigau et al. 2003). A highly interesting idea was raised by Liebert \& Burgasser (2007) who suggested that the emission could be caused by a smaller companion with $T_{\text {eff }}<500 \mathrm{~K}$ based on a possible color excess seen in Spitzer IRAC photometry. Important constraints on 2MASS J1237+6526 stem from radio observations by Kao et al. (2016, 2018). The radio emission includes a highly circularly polarized and pulsed component, which was used to derive the dwarf's rotation period of $2.28 \mathrm{~h}$ (Kao et al. 2018). The radio emission provides evidence that 2 MASS $\mathrm{J} 1237+6526$ possesses a very strong average surface magnetic field of at least $2.9 \mathrm{kG}$. In analogy with the circularly polarized radio emission from the aurorae of the Solar System planets, Kao et al. (2016, 2018) hypothesize that the circularly polarized radio emission from 2MASS J1237+6526 may be auroral emission. However, it remains unclear whether this emission is really caused by auroral electrons that are energized outside of the dwarf's atmosphere, chromosphere, or ionosphere (i.e., in the dwarf's magnetosphere).

In this paper we first discuss properties of the plasma and magnetic field environment around brown dwarfs, planets, and low mass stars in a broader context, and derive scaling models for their potential auroral luminosities (Sect. 2.3). Then we present the analysis of HST observations of the brown dwarf 2MASS J1237+6526 (Sect. 3). Subsequently, we discuss what the HST observations of 2MASS J1237+6526 might imply in the context of its possible auroral emission (Sect. 4).

\section{Auroral power potential}

Aurorae are defined as electromagnetic emission caused by impacting electrons or ions on atmospheric neutrals, where the impacting particles are produced outside the objects' atmospheres (i.e., usually in their magnetospheres). In this section we derive estimates of potential auroral powers based on the underlying energetics of magnetospheric processes, which are the root cause of auroral emission. We show that brown dwarfs are ideal objects to search for UV aurorae outside the Solar System.

\subsection{Auroral power: overview}

Auroral processes require three components: (1) a generator that produces electric current and electromagnetic power, (2) an accelerator where the electromagnetic energy is converted into particle acceleration, and (3) an atmosphere where the energized particles precipitate into and excite auroral emission (e.g., Mauk \& Bagenal 2012).

The generators known in the Solar System are powered in one of three different ways: (i) driven by plasma flow external to the planet's magnetospheres, (ii) driven by plasma transport within the planets' fast rotating magnetospheres, or (iii) driven by moons within the planets' magnetospheres.

The aurora of the Earth falls into category (i) and possesses a solar wind driven aurora, where the energy ultimately comes from the solar wind flow exerting forces on Earth's magnetosphere and driving reconnection at the magnetopause (e.g., Mauk \& Bagenal 2012). Similarly, Ganymede's aurora is mostly powered by the flow of plasma from Jupiter's magnetosphere against Ganymede's mini-magnetosphere (e.g., Eviatar et al. 2001).

Jupiter's main auroral emission is a prime example of category (ii), an aurora driven by internal magnetospheric plasma transport. In the case of Jupiter, its close-in moon Io is the root cause of plasma production at a rate of about 1 ton/s (e.g., Broadfoot et al. 1979; Hill 1979; Dessler 1980; Smith \& Strobel 1985). Jupiter's fast rotation (with a period of about $10 \mathrm{hr}$ ) generates strong centrifugal forces within its magnetosphere, which cause the magnetospheric plasma to move radially outward. Due to conservation of angular momentum the corotation of the outward moving plasma breaks down with distance, which means that the magnetospheric plasma does not fully corotate 
with Jupiter any more. The sub-corotating plasma thus generates magnetic stresses that couple Jupiter's magnetosphere to its ionosphere. This coupling drives angular momentum and energy transport between the two regions, which spins up the magnetosphere toward corotation, but slows down the angular velocity of its ionosphere. In the case of Jupiter this process is the main auroral power generator (Hill 2001) even though many details of the coupling and the physics of the particle acceleration are currently being investigated via Juno spacecraft measurements (e.g., Mauk et al. 2017; Clark et al. 2018; Saur et al. 2018b). The underlying mechanism of how a rotating and expanding magnetosphere couples to its ionosphere is similar to magnetic braking where an expanding stellar wind magnetically couples to its star and subsequently slows down the rotation of the star.

Aurorae of category (iii) are caused by moons within planetary magnetospheres. The moons are obstacles to the magnetospheric plasma and perturb the plasma flow and magnetic field. This also causes magnetic stresses, which propagate along the planet's magnetic field and carry high energy fluxes between the moons and their host planets (Goldreich \& Lynden-Bell 1969; Neubauer 1980; Goertz 1980; Zarka 2007; Saur et al. 2013).

In the following we only consider auroral processes (ii) and (iii), those due to magnetospheric mass transport and a planetary companion. The first process (i), flow external to the magnetosphere, is not expected to be relevant for brown dwarfs, due to the expected very large sizes of brown dwarf magnetospheres. The sizes of their magnetospheres are not known from direct observations, nor are the detailed properties of the interstellar medium (ISM) surrounding the brown dwarfs. To estimate the sizes of the dwarfs' magnetospheres, we assume ISM properties similar to those near the heliosphere from McComas et al. (2012) with a magnetic field strength of $B_{\mathrm{ISM}}=3 \times 10^{-6}$ gauss, a proton density $n_{\mathrm{ISM}}$ of $0.07 \mathrm{~cm}^{-3}$, and a relative velocity between the heliosphere and the ISM of $v_{\text {ISM }}=23.2 \mathrm{~km} \mathrm{~s}^{-1}$. We assume that the magnetic pressure of the dwarf's magnetosphere is balanced by the sum of the magnetic and ram pressure of the ISM at the magnetopause. Under the assumption of a dipole field for the brown dwarf, this balance leads to a location of the magnetopause in units of the brown dwarf's radius $R_{\mathrm{BD}}$ given by

$$
\frac{r}{R_{\mathrm{BD}}}=\left(\left(\frac{B_{\mathrm{ISM}}}{B_{\mathrm{BD}}}\right)^{2}+\left(\frac{m_{\mathrm{p}} n_{\mathrm{ISM}} v_{\mathrm{ISM}}^{2}}{B_{\mathrm{BD}}^{2} / 2 \mu_{0}}\right)\right)^{-1 / 6},
$$

with $m_{\mathrm{p}}$ the proton mass, $\mu_{0}$ the permeability of free space, and $B_{\mathrm{BD}}$ the equatorial field strength of the brown dwarf. This expression yields a magnetopause distance of $740 R_{\mathrm{BD}}$ for 2 MASS J1237+6526. Any flow of plasma inside the magnetosphere will shift the location of the magnetopause farther away. The estimated gigantic size of the dwarf's magnetosphere can be assumed to be typical for strongly magnetized brown dwarfs. The size implies that the interaction of the interstellar medium with this magnetosphere and similar dwarf magnetospheres occur at such large distances that auroral coupling processes to the dwarfs' atmospheres are likely ineffective.

\subsection{Auroral power: scaling models}

In this subsection we derive scaling models for luminosities of aurorae driven by magnetospheric mass transport or a planetary companion. These scaling models are applicable to the various wavelength ranges employed in the search and characterization of exo-aurorae. We show that it is possible to introduce a universal quantity, referred to as auroral power potential $S_{\text {pot }}$, which characterizes the ability of an object to generate aurora. The subsequent models assume for mathematical simplicity a pure dipole magnetic field for the primary bodies, even though the planets in the Solar System and low mass stars are known to possess magnetic fields with higher-order moments (e.g., Connerney et al. 2018; Morin et al. 2008b; Yadav et al. 2015; Berdyugina et al. 2017). Magnetic field contributions from higher-order moments fall off more rapidly with distance than dipole components, thus at the magnetospheric locations of the auroral generators the dipole components likely dominate the local magnetic fields. In this work the atmospheres of brown dwarfs are assumed to possess electrically conductive layers similar to the atmospheres of the planets in the Solar System (e.g., Rees 1989). Such ionized layers on brown dwarfs can form via the interstellar radiation field or ionizing auroral electrons beams, for example, and have also been referred to as ionospheres on brown dwarfs (Helling \& Rimmer 2019). These ionized layers provide free electrons and ions that can drive magnetospheric current systems and/or chromospheric heating (Rodríguez-Barrera et al. 2018).

\subsubsection{Aurora due to magnetospheric mass transport}

Hill (2001) derived an expression, resulting from the mass transport in Jupiter's fast rotating magnetosphere, which characterizes the energy flux between the magnetosphere and its ionosphere. This energy flux is given by expression (4) in Hill (2001) and reads

$P_{\mathrm{mag}, J}=2 \pi \Sigma_{J} B_{J}^{2} \Omega_{J}^{2} R_{J}^{4} / \hat{L}_{J}^{2}$,

with Jupiter's dipolar equatorial surface magnetic field $B_{J}$, its angular velocity $\Omega_{J}$, the ionospheric conductance $\Sigma_{J}$, Jupiter's radius $R_{J}$, and the distance $\hat{L}_{J}$ between Jupiter and the magnetospheric region where the corotation breaks down. The $\hat{L}$ parameter is dimensionless and describes the breakdown distance in units of $R_{J}$. In Jupiter's magnetosphere the breakdown occurs around $\hat{L}_{J} \sim 30$ (Hill 2001). At the distance $\hat{L}_{J}$, the energy flux between the magnetosphere and the ionosphere maximizes. We generalize expression (2) to an arbitrary host body with similar magnetospheric transport processes and rewrite the expression as

$P_{\text {mag }}=S_{\text {pot }}\left(\pi \frac{R_{\text {host }}^{2}}{\hat{L}_{\text {host }}^{2}}\right) \Sigma_{\text {host }}$,

where the variables with the subscript "host" refer to the auroral host object under consideration. The host can be a planet, a brown dwarf, or a star. In expression (3) we combined the quantities polar magnetic field strength $B_{\text {host }}$, angular velocity $\Omega_{\text {host }}$, and radius $R_{\text {host }}$ of the auroral emitting host to a new quantity, which we refer to as auroral power potential:

$S_{\text {pot }}=B_{\text {host }}^{2} \Omega_{\text {host }}^{2} R_{\text {host }}^{2}$.

This quantity is universal for the cases studied here, meaning that it characterizes auroral power generated by magnetospheric mass transport and by a companion, as shown in this Subsection and in Sect. 2.2.2, respectively. The auroral power potential can be written as $B_{\text {host }}^{2} v_{\text {host }}^{2}$ with the velocity given by $v_{\text {host }}=\Omega_{\text {host }} R_{\text {host }}$. Assuming the frozen-in field theorem $\mathbf{E}=-\mathbf{v} \times \mathbf{B}$ with $\mathbf{E}$ the motional electric field in the nonrotating rest frame, the auroral power potential is simply $E_{\text {host }}^{2}$ and therefore proportional to the work per volume element $\mathbf{j}_{\text {host }} \cdot \mathbf{E}_{\text {host }}=\sigma_{\text {host }} E_{\text {host }}^{2}$ with the conductivity $\sigma_{\text {host }}$ of the host's ionosphere. Thus, the auroral power potential is the power that can be exerted by the flow $v_{\text {host }}$ in a 
magnetic field $B_{\text {host }}$ per unit conductivity and unit volume on the surface of the host. The total power in Eq. (3) is then given by integration of the power potential over the volume on the host where the auroral coupling and emission occurs multiplied by its conductivity. The conductivity $\sigma$ integrated along the magnetic field lines, which are essentially radial within the planet's polar ionosphere, leads to the conductances $\Sigma_{\text {host }}$. Thus, expression (3) can be rewritten as

$P_{\text {mag }}=S_{\text {pot }} \underbrace{\Sigma_{\text {host }} A_{\text {auroral }} \hat{v}^{2}}_{\mathrm{Q}}$,

with the area $A_{\text {auroral }}$ on the planet from which the auroral emission occurs. In this model it is located at colatitude $\Theta$ given by $\sin \Theta=\hat{L}^{-1 / 2}$ (Hill 2001). It would correspond to a width of $\Delta \Theta=\frac{1}{4} \hat{L}^{-1 / 2}$ of the auroral ovals. In units of degrees the location of Jupiter's auroral oval is thus at $10.5^{\circ}$ colatitude with a width of $2.6^{\circ}$ (based on $\hat{L}=30$ ). Since the location of the oval is at $\Theta$, the velocity $v$ of the ionosphere is not $R_{\text {host }} \Omega$, but reduced by a dimensionless quantity, $\hat{v}=v(\Theta) /\left(R_{\text {host }} \Omega_{\text {host }}\right)=\sin \Theta$. We combine the last three terms in Eq. (5) to a new quantity $\mathrm{Q}$, which represents the individual characteristics of the auroral driver within a host's magnetosphere. Thus, in simple words, the total power from which the aurora at various wavelengths draws its power is given by the auroral power potential $S_{\text {pot }}$ times a factor $\mathrm{Q}$ which depends on the details of each magnetosphere under consideration. The Q-factor is expected to vary individually and can be different for magnetospheres with even the same power potential. The Q-factor depends, for example, on the plasma sources and their composition, the mass transport in the magnetosphere, and the ionization state of the hosts.

It is instructive to compare the electromagnetic energy fluxes derived here assuming the ionosphere of the host can be characterized by a spinning disk with angular velocity $\Omega_{\text {host }}$, radius $R_{\text {host }}$, and conductivity $\sigma$ within a magnetic field $B_{\text {host }}$ parallel to the spin axis of the disk. The work done per unit time $\mathbf{j} \cdot \mathbf{E}$ within the whole conducting disk is given by

$P_{\text {disk }}=\frac{1}{2} \pi \Sigma_{\text {host }} R_{\text {host }}^{2} S_{\text {pot }}$.

The energy flux in Eq. (5) can be written in units of the work done by a disk as

$P_{\text {mag }}=2 \hat{A}_{\text {auroral }} \hat{v}^{2} P_{\text {disk }}$,

with $\hat{A}_{\text {auroral }}$ the size of the auroral area in each hemisphere normalized to the size of the disk and $\hat{v}$ the normalized rotation velocity of the disk at the location of the auroral area.

The expressions Eqs. (3), (5), or (7) describe in various ways the total energy flux between the host and its magnetosphere due to radial mass transport. Only a fraction of this energy flux is converted into particle acceleration and subsequently into auroral emission at a certain wavelength or wavelength band. We characterize this fraction by an efficiency factor $\epsilon$. In the case of Jupiter the total energy flux between its ionosphere and magnetosphere has been estimated as $P_{\text {mag }}=3.1 \times 10^{14}$ watt by Hill (2001). Jupiter's auroral energy fluxes within different wavelength bands and the associated fraction $\epsilon_{J}$ are given in Table 1 . We thus can write the auroral luminosity for a certain wavelength range as

$L_{\text {mag }}=S_{\text {pot }}\left(\pi \frac{R_{\text {host }}^{2}}{\hat{L}_{\text {host }}^{2}}\right) \Sigma_{\text {host }} \epsilon_{\text {host }}$.
Alternatively, it is useful to write the auroral luminosity in Eq. (8) as a scaling model with respect to observed auroral luminosities of the Jupiter system $L_{\mathrm{mag}, J}$ within certain wavelength bands as

$$
L_{\text {mag }}=\underbrace{\left(\frac{S_{\text {pot, host }}}{S_{\text {pot }, J}}\right)}_{S_{\text {pot,rel }}} \underbrace{\left(\frac{\Sigma_{\text {host }}}{\Sigma_{J}} \frac{\hat{L}_{\text {host }}^{-2}}{\hat{L}_{J}^{-2}} \frac{R_{\text {host }}^{2}}{R_{J}^{2}} \frac{\epsilon_{\text {host }}}{\epsilon_{J}}\right)}_{Q_{\text {rel }}} L_{\text {mag,J },}
$$

where the subscript $J$ refers to values for Jupiter with $S_{\text {pot }, J}=600$ watt $^{-2}$ siemens $^{-1}$ based on the values from Table A.1. Values for Jupiter's ionospheric conductances $\Sigma_{J}$ from the literature lie around 1 siemens (e.g., 0.6 siemens in Hill 2001 or 5 siemens in Strobel \& Atreya 1983 and $\hat{L}_{J}=30$, Hill 2001). It should be noted that in this work $\hat{L}$ refers to the normalized location of corotation break down, while other variables $L$ without a "hat" refer to the various expressions for luminosities. The first term on the right-hand side describes the power potential relative to Jupiter $S_{\text {pot,rel }}$. The other quantities within the second set of parentheses combined as $Q_{\text {rel }}$ in Eq. (9) describe auroral generator characteristics relative to Jupiter. $S_{\text {pot }}$ and $Q_{\text {rel }}$ are independent, which means that a host can have in principle a larger auroral power potential, but in the absence of a magnetospheric plasma source $\mathrm{Q}$ can still be zero. The $\mathrm{Q}$ factor includes the location where the corotation breaks down $\left(\hat{L}_{\text {host }}\right)$, the ionospheric conductance $\left(\Sigma_{\text {host }}\right.$, i.e., the altitude integrated conductivity $\sigma$ ), and the efficiency $\left(\epsilon_{\text {host }}\right)$, which all depend on the details of the mass sources within the magnetosphere and the ionospheric properties of the host system. Possible ionization can come from external ionizing UV radiation or internal impact ionization, which depend on the individual system (RodríguezBarrera et al. 2018). Aside from the radius, which for brown dwarfs can be assumed to be around Jupiter-size, the other parameters of $\mathrm{Q}$ are difficult to estimate without further information. Observations of auroral emission, however, will allow us to constrain them.

\subsubsection{Aurora due to a planetary companion}

In case of a planetary companion within the host's magnetosphere, the associated auroral processes can be described by two different models, depending on the plasma density in the magnetosphere. For very low densities and nearly unpopulated magnetospheres, the coupling between the planetary companion and the central body is given by the unipolar inductor model (Goldreich \& Lynden-Bell 1969); for higher densities the coupling turns into the Alfvén wing model (Neubauer 1980; Goertz 1980; Saur 2004). The conditions for the transitions between the two models are discussed in detail in Neubauer (1998). It is unclear whether magnetospheres of brown dwarfs are filled with plasma or are mostly empty. The calculations below show that a companion in either case will generate an electromagnetic coupling and auroral phenomena on the host body.

The energy flux in the unipolar inductor model was derived by Goldreich \& Lynden-Bell (1969) as

$P_{\text {uni }}=\pi \Sigma_{J} E_{J}^{2}(X+Y) Y$,

with the motional electric field $E_{J}$ and $\mathrm{X}$ and $\mathrm{Y}$ the semimajor and semiminor axes of the resulting electromagnetic flux tube originating between Io and Jupiter with the values taken at the surface of Jupiter. We can rewrite Eq. (10) and generalize it to an arbitrary companion orbiting a host. We also introduce an 
Table 1. Auroral luminosities at Jupiter and efficiency factor for various wavelength bands.

\begin{tabular}{lrrr}
\hline \hline Wavelength & $\begin{array}{r}\text { Luminosity } L_{\text {mag }} \\
\text { main oval [watt] }\end{array}$ & $\begin{array}{r}\text { Luminosity } L_{\text {comp }} \\
\text { Io footprint [watt] }\end{array}$ & Efficiency $\epsilon$ \\
\hline X-ray & $1-4 \times 10^{9}(\mathrm{a})$ & & $0.3-1.3 \times 10^{-5}$ \\
Far-UV & $2-10 \times 10^{12}(\mathrm{a})$ & $0.4-30 \times 10^{10}(\mathrm{c})$ & $0.6-3 \times 10^{-2}$ \\
Near-UV & $2-10 \times 10^{11}(\mathrm{a})$ & $\sim 5 \times 10^{9}(\mathrm{a})$ & $0.6-3 \times 10^{-3}$ \\
Visible & $1-10 \times 10^{10}(\mathrm{a})$ & $\sim 3 \times 10^{8}(\mathrm{a})$ & $0.3-3 \times 10^{-4}$ \\
IR & $\sim 50 \times 10^{12}(\mathrm{a})$ & $3-10 \times 10^{10}(\mathrm{a})$ & $\sim 10^{-1}$ \\
Radio & $1-10 \times 10^{10}(\mathrm{a}, \mathrm{b})$ & $1-100 \times 10^{8}(\mathrm{a}, \mathrm{d})$ & $0.3-3 \times 10^{-4}$ \\
\hline
\end{tabular}

Notes. The X-ray scaling needs to be taken with caution as the origin of Jupiter's X-ray emission is not fully understood. References. (a): Bhardwaj \& Gladstone (2000), (b): Zarka (1998), (c) Wannawichian et al. (2010), (d) Zarka (2007).

efficiency factor $\epsilon_{\text {host }}$ for converting the energy flux into auroral emission within a specific wavelength band. This leads to

$L_{\mathrm{uni}}=S_{\mathrm{pot}}\left(\frac{3 \pi}{4} \frac{R_{\mathrm{comp}}^{2}}{\hat{a}_{\mathrm{comp}}^{4}} \Sigma_{\text {host }} \epsilon_{\mathrm{host}}\right)$,

with the radius $R_{\text {comp }}$ of the companion and the distance $\hat{a}_{\text {comp }}$ between the planet and the companion in units of the radius of the host body $R_{\text {host }}$. The values for $\epsilon$ for the auroral imprint of Io in Jupiter's atmosphere are similar to those of Jupiter's main aurora (based on Bhardwaj \& Gladstone 2000).

The expression for the energy flux within the Alfvén wing model was derived by Saur et al. (2013) as

$P_{\mathrm{Alf}}=2 \pi R_{\mathrm{Io}}^{2} \frac{E_{\mathrm{Io}} B_{\mathrm{Io}}}{\mu_{0}} M_{\mathrm{A}}$,

with the motional electric field $E_{\mathrm{Io}}$ and Jupiter's magnetospheric field strength $B_{\mathrm{Io}}$, both at the location of Io, the radius $R_{\mathrm{Io}}$ of Io, the permeability of free space $\mu_{0}$ and the Alfvén Mach number $M_{\mathrm{A}}$, i.e., the ratio of the flow velocity $v$ to the Alfvén velocity $v_{\mathrm{A}}$ in Jupiter's magnetospheric plasma at the location of Io. In expressions (10) and (12) it is assumed that Io possesses a highly electrically conductive ionosphere. We again can generalize the expression (12) to an arbitrary companion and host and calculate the resultant luminosity $L_{\text {Alf }}$

$L_{\text {Alf }}=S_{\text {spot }}\left(\frac{\pi}{2} \frac{R_{\text {comp }}^{2}}{\hat{a}_{\text {comp }}^{4}}\right) \Sigma_{\mathrm{A}} \epsilon_{\text {host }}$,

with an auroral efficiency factor $\epsilon_{\text {host }}$ and Alfvén conductance $\left.\Sigma_{\mathrm{A}}=1 /\left(\mu_{0} v_{\mathrm{A}}\right)=\sqrt{(} \rho \mu_{0}\right) / B$, with the magnetospheric field strength $B$, and the mass density $\rho$ at the location of the companion. In the case of Jupiter, $\Sigma_{\mathrm{A}}$ and $\Sigma_{\text {host }}$ assume comparable values (Strobel \& Atreya 1983). In this derivation for simplicity we neglect the angular velocity of the companion compared to the usually significantly higher angular velocity of the fast rotating planets or brown dwarfs. This however could easily be fixed by replacing $\Omega$ with the relative angular velocity between the rotation of the host and the angular velocity of the companion. The radius of the companion is its effective radius (i.e., the radius including the companion's atmosphere and ionosphere). When the companion possesses an internal magnetic field, the effective radius can be significantly enhanced (e.g., Neubauer 1998; Saur et al. 2013), up to $\sqrt{3} R_{\mathrm{M}}$ with $R_{\mathrm{M}}$ being the radial distance of the companion's magnetopause. In this case, reconnection at the companions magnetopause generates electrical conductivity of the object.
The energy fluxes induced by a companion in the unipolar inductor case (10) and Alfvén wing case (12) can jointly be written as

$$
\begin{aligned}
P_{\text {comp }} & =\gamma \pi \Sigma R_{\text {comp }}^{2} \hat{a}^{-4} S_{\text {pot }} \\
& =2 \gamma \Sigma \hat{v}^{2} A_{\text {eff }} S_{\text {pot }} \\
& =4 \gamma \hat{v}^{2} \hat{A}_{\text {eff }} P_{\text {disk }}
\end{aligned}
$$

with $\gamma=3 / 4$ for the unipolar inductor case and $\gamma=1 / 2$ for the Alfvén wing case. The conductance $\Sigma$ may represent either the conductance of the host body's ionosphere $\Sigma_{\text {host }}$ in the unipolar inductor case or the Alfvén conductance $\Sigma_{\mathrm{A}}$. The parameter $A_{\text {eff }}$ is the effective area of the companion mapped along the host's magnetic field line onto the host, and $\hat{A}_{\text {eff }}$ is $A_{\text {eff }}$ normalized to $\pi R_{\text {host }}^{2}$. Expressions in (14) show why it is better to characterize the ability of a host to exhibit aurora with a local quantity such as $S_{\text {pot }}$ on the host rather than a global quantity such as the power of a rotating disk $P_{\text {disk }}$. Hosts with different sizes but the same power potential would lead to the incorrect impression that they will produce different auroral emissions for companions with the same properties.

The resulting luminosities for the unipolar inductor and the Alfvén wing model can now also be generalized to those caused by an arbitrary companion within a rotating magnetosphere. Using (14), we find for the auroral luminosities caused by a companion in units of the auroral luminosity $L_{\mathrm{Io}}$ caused by Io

$$
L_{\text {comp }}=\underbrace{\left(\frac{S_{\text {pot, host }}}{S_{\text {pot, } \mathrm{J}}}\right)}_{S_{\text {pot,rel }}} \underbrace{\left(\frac{\Sigma}{\Sigma_{\mathrm{J}}} \frac{R_{\mathrm{comp}}^{2}}{R_{\mathrm{Io}}^{2}} \frac{a_{\mathrm{comp}}^{-4}}{a_{\mathrm{Io}}^{-4}} \frac{\epsilon_{\mathrm{host}}}{\epsilon_{\mathrm{J}}}\right)}_{Q_{\text {rel }}} L_{\mathrm{Io}} .
$$

The luminosity due to a companion thus can also be written as the product of the power potential of the host relative to the one of Jupiter $S_{\text {pot,rel }}$ and a relative $Q_{\text {rel }}$ factor considering the properties of the companion. The auroral luminosities caused by Io in Jupiter's atmosphere are displayed in Table 1. Io's induced emission depends on its position in Jupiter's magnetosphere, which leads to the range of parameters in the table. The efficiency factors for Io's auroral footprint are similar to those of the main oval based on the theoretically expected fluxes of 300$2000 \times 10^{9}$ watt from Saur et al. (2013), and therefore are not shown separately in Table 1 .

Expressions (9) and (15) show that the power for both auroral processes (i.e., magnetospheric transport or a companion) is proportional to the quantity $S_{\text {pot,host }}$, which describes the potential for an object to exhibit auroral emission. The terms in the second 


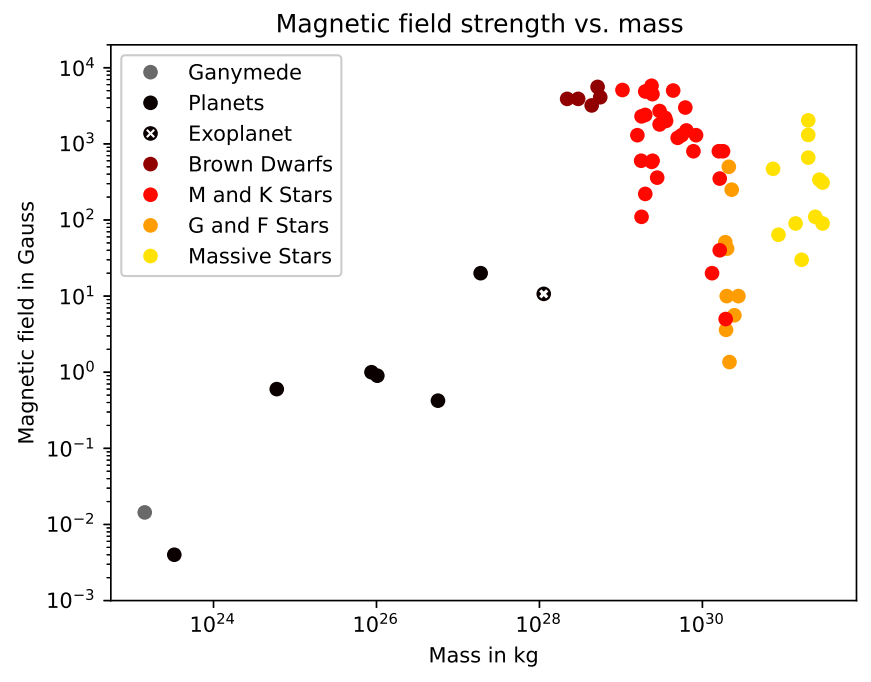

Fig. 1. Observed surface magnetic field strengths or lower limits of surface fields (depending on observation method, see Appendix A) as a function of mass for selected planetary bodies, brown dwarfs, and stars. The names of the objects with further details and associated references are provided in Appendix A.

set of parentheses, the Q-factor, contains the properties of the magnetospheric mass transport, the size and the distance of the companion plus its local plasma environment, and the efficiency factor $\epsilon$.

In the next subsection we investigate the effects of the auroral power potential relative to Jupiter for objects where $\Omega, B$, and $R$ are known. This gives a guideline for selecting target objects to detect auroral emission. Measurements of auroral fluxes from these objects will be a powerful tool to constrain individual properties of the system included in the parameter Q (Eqs. (9) and (15)).

\subsection{Auroral power potential: brown dwarfs, planets, and stars}

To investigate the role of the auroral power potential $S_{\text {pot }}$ we selected a number of bodies covering the mass range of planets, brown dwarfs, and stars (see Table A.1). Significant auroral emission in the Solar System only occurs on planetary bodies with intrinsic magnetic fields. Intrinsic dynamo fields exist for Mercury, Earth, Jupiter, Saturn, Uranus, and Neptune and for Jupiter's moon Ganymede. Recently, a tentative detection of a magnetic field on the exoplanet $\tau$ Boötis b based on radio observations with LOFAR by Turner et al. (2021) has been reported, which we also include. For these planetary bodies, together with brown dwarfs and low to intermediate mass stars, their magnetic field strength as a function of the object's mass is displayed in Fig. 1. The magnetic fields have been determined with various methods, such as in situ measurements for the Solar System targets, observations of radio emission near the electron cyclotron frequency for brown dwarfs and $\tau$ Boötis $b$, or with methods based on the Zeeman-effect for the stars (see last column in Table A.1 for the explicit method applied for each target). Some of these values might be lower limits as radio emission, for example, often stems from magnetospheric layers above the objects' surfaces. Despite these different techniques, Fig. 1 still provides a general overview of the magnetic field strength for the various classes of objects. We include a wide spread of masses from small planetary objects to high mass stars for a basic visualization of the overall field strength distribution. The

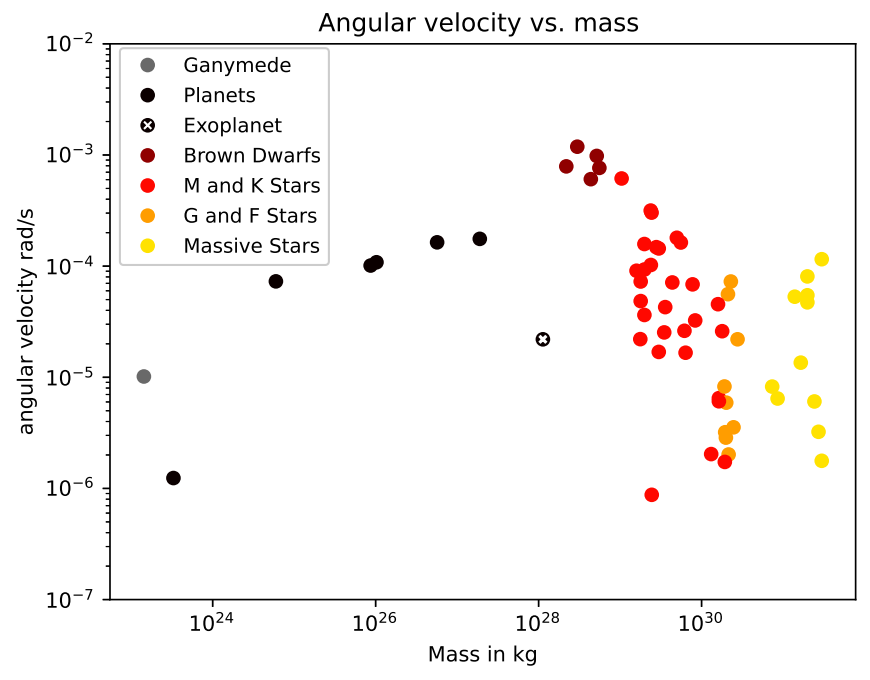

Fig. 2. Angular velocity as a function of mass for selected magnetized planetary bodies, brown dwarfs, and stars. The names of the objects with further details and associated references are provided in Appendix A.

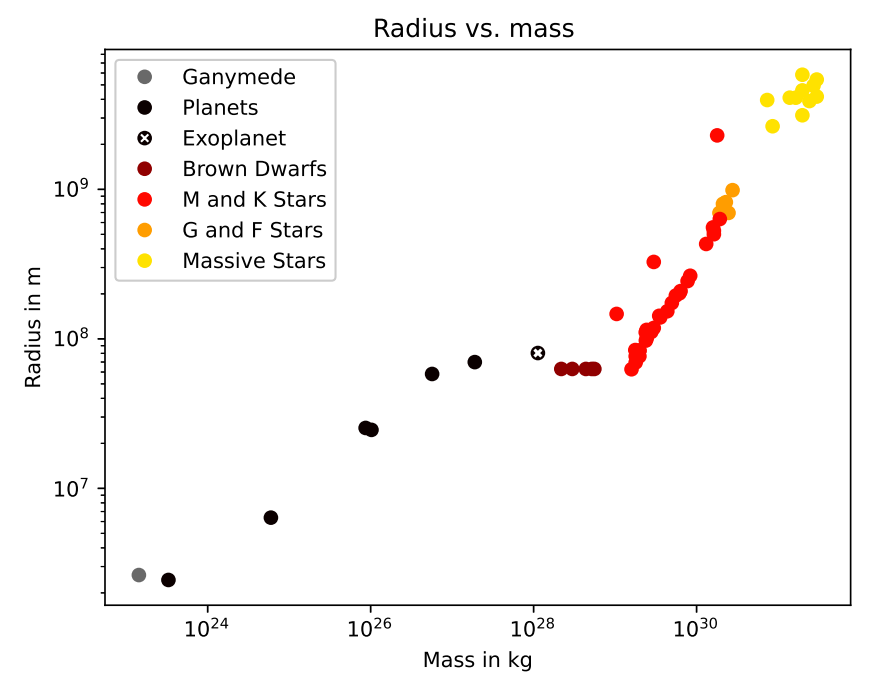

Fig. 3. Radius as a function of mass for selected magnetized planetary bodies, brown dwarfs, and stars. The names of the objects with further details and associated references are provided in Appendix A.

largest magnetic fields typically occur within the brown dwarf mass range into the low mass star regime, at least for the cases where magnetic fields have been observed. Similarly the rotation periods of brown dwarfs are typically very short, on the order of hours (Pineda et al. 2017; Tannock et al. 2021), which results in the high angular velocities in the brown dwarf mass regime displayed in Fig. 2. The radii of the objects on average grows with mass, as displayed in Fig. 3. However, in the brown dwarf mass regime the radius-mass relation is degenerated due to gravitational forces that alter the balance between electrostatic attraction and electron degeneracy pressure.Thus, brown dwarf radii are fairly constant at values of about $0.9 R_{\mathrm{J}}$ (Vrba et al. 2004; Kao et al. 2016; Hatzes \& Rauer 2015).

With the magnetic field, the angular velocity, and the radius from each body in Table A.1, we now calculate the auroral power potential using expression (4) for the selected bodies. The results are displayed in Fig. 4. The auroral power potential shows a 


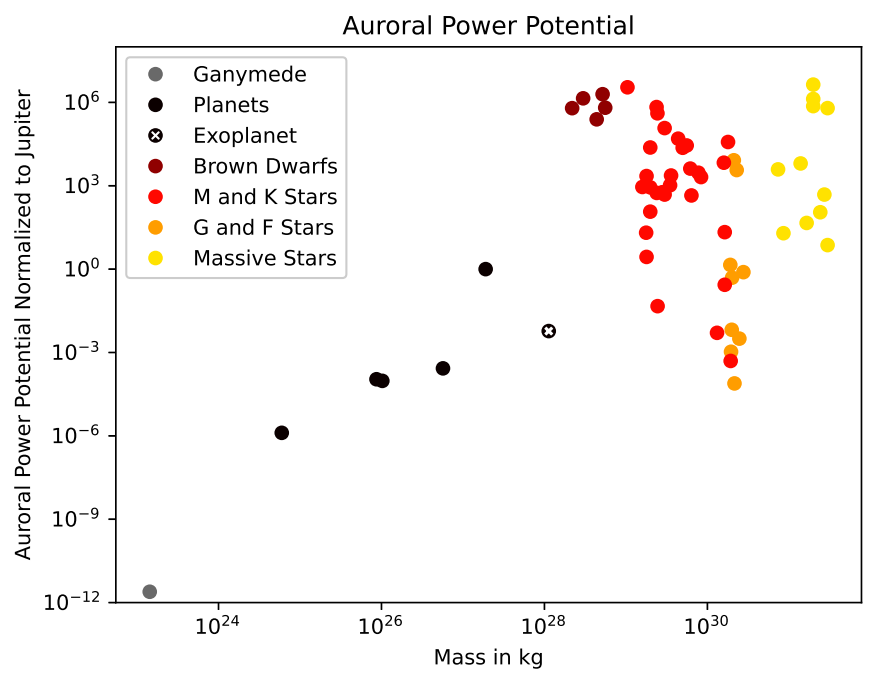

Fig. 4. Auroral power potential normalized to the auroral power of Jupiter for aurora types that are driven by radial mass transport within strongly rotating magnetospheres or by planetary companions (see text and Appendix A).

maximum for brown dwarfs and very low mass stars. It can be six orders of magnitude larger than Jupiter. Jupiter's auroral UV luminosity is in the range of $2-10 \times 10^{12}$ watt (Bhardwaj \& Gladstone 2000). Thus, brown dwarfs have the potential for UV luminosities on the order of $10^{19}$ watt. The real auroral powers however can be different, higher or lower, as they additionally depend on the properties included in the $Q_{\text {rel-factors in expres- }}$ sions (9) and (15). B stars tend to have large auroral power potential values similar to those of brown dwarfs due to their particularly large radii (Fig. 3).

The auroral power potentials can be compared with the real auroral powers of the planets and the moon Ganymede. The three lowest mass objects are of terrestrial type: the moon Ganymede, and the planets Mercury and Earth. Mercury does not exhibit aurora because it does not possess an atmosphere. Ganymede's auroral emission has been observed in the FUV in the oxygen lines O I 1304 and $1356 \AA$ (e.g., Hall et al. 1998; Feldman et al. 2000; Saur et al. 2015). Based on the measured fluxes, we can derive a FUV luminosity of $3 \times 10^{7}$ watt, i.e., a factor of $\sim 10^{5}$ weaker than Jupiter's. Earth's auroral luminosity is a factor of about $10^{3}$ lower than that of Jupiter (Bhardwaj $\&$ Gladstone 2000). However, the aurorae of these objects are driven by reconnection and plasma flow outside of their respective magnetosphere, and thus do not fall into the category driven by internal mass flow or driven by a companion.

The outer planets Jupiter, Saturn, Uranus, and Neptune are all fast rotators, possess magnetic fields, and exhibit auroral emissions. Saturn's auroral UV power is $50 \times 10^{9}$ watt, that of Uranus $40 \times 10^{9}$ watt, Neptune's only $0.1 \times 10^{9}$ watt (Bhardwaj \& Gladstone 2000). The auroral UV luminosities of Saturn and Uranus are thus $10^{2}$ to $10^{3}$ times smaller compared to that of Jupiter. This fits well into our estimates shown in Fig. 4 in particular given that they are somewhat lower compared to Jupiter. The low UV luminosity of Neptune, despite its auroral potential, is generally attributed to a very weak generator (i.e., very low mass transport rates in the Triton torus) (Bhardwaj \& Gladstone 2000).

\subsection{Thermal UV emission}

Brown dwarfs are particularly well suited targets to search for UV aurora outside the Solar System because, in addition to their

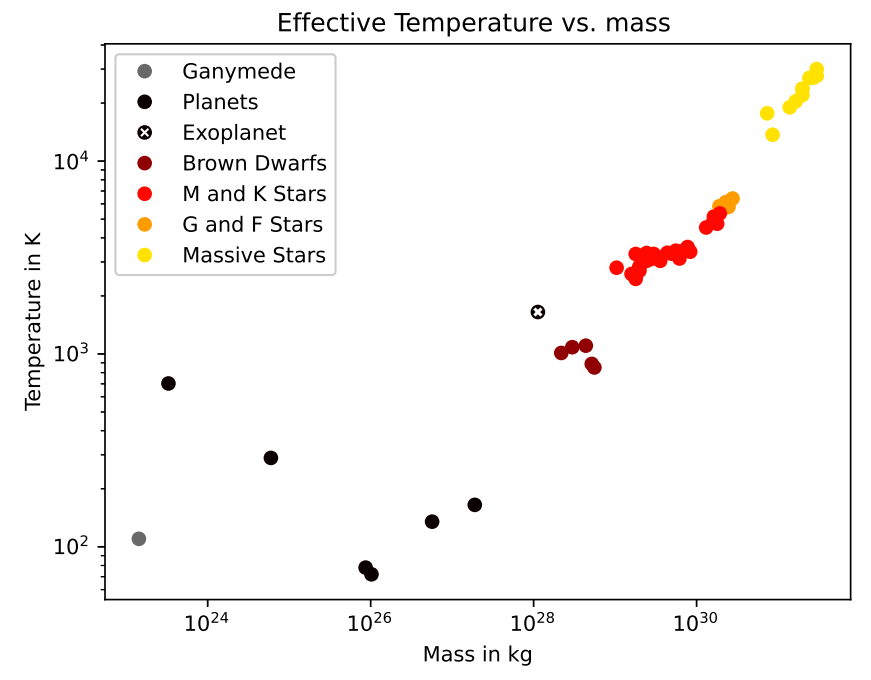

Fig. 5. Effective temperatures of selected magnetized objects (see Table A.1) as a function of mass.

large auroral power potential, they exhibit low effective temperatures. In Fig. 5 we display the effective temperatures of the objects spanning the mass range from planetary objects to massive stars. The surface temperatures of Ganymede and the planets are primarily controlled by solar irradiance and the thermal IR emission. Effective temperatures of Y- to L-type brown dwarfs lie in the range $250-2100 \mathrm{~K}$, while $\mathrm{M}$ dwarfs, which are usually low mass stars, lie in the range 2100-3500 K (e.g., Filippazzo et al. 2015; Luhman 2014). Solar-type stars possess effective temperatures in the range of $5000 \mathrm{~K}$ to nearly $10,000 \mathrm{~K}$ and massive stars temperatures well above 10,000 K (references for each object are provided in Table A.1).

The expected thermal UV power strongly decreases with temperature of stars and brown dwarfs, and thus also roughly decreases with mass. The T6.5 dwarf 2MASS J1237+6526 possesses a temperature of $830 \mathrm{~K}$ (Kao et al. 2016). Assuming only thermal emission from the target we would expect a total luminosity of $10^{2}$ watt in the NUV within 1570-3180 $\AA$, and a total luminosity of $10^{-16} \mathrm{~W}$ in the FUV within $1150-1730 \AA$. Both energy fluxes are entirely negligible compared to the potential auroral UV emission of $10^{19} \mathrm{~W}$ from the dwarf.

To further illustrate the competing effects of thermal UV emission compared to possible auroral emission, we display in Fig. 6 the thermal FUV and thermal NUV for each object assuming a blackbody emission with the effective temperatures from Fig. 5. Due to the highly nonlinear temperature dependence in Planck's law the thermal luminosity varies over more than 40 orders of magnitudes from the brown dwarf to the massive star regime. The thermal UV emission from the outer planets and Ganymede is so small that it is below the displayed range. Only Mercury, due to its proximity to the sun, is within the plot range of Fig. 6. In comparison to the thermal UV luminosities, we show possible FUV luminosities applying expression (9) with $Q_{\text {rel }}=1$ and the average FUV luminosity of Jupiter $L_{\text {mag,J }}$ from Table 1. We do not show the NUV auroral luminosity because it is a constant factor of 10 lower than the FUV and would only overcrowd the figure.

Figure 6 clearly shows that for the outer planets and brown dwarfs the thermal UV is orders of magnitude below possible auroral UV luminosities. For early-type M-dwarf stars and more massive K-type stars the thermal UV emission starts to 
Potential auroral vs thermal luminosities for NUV and FUV wavelengths

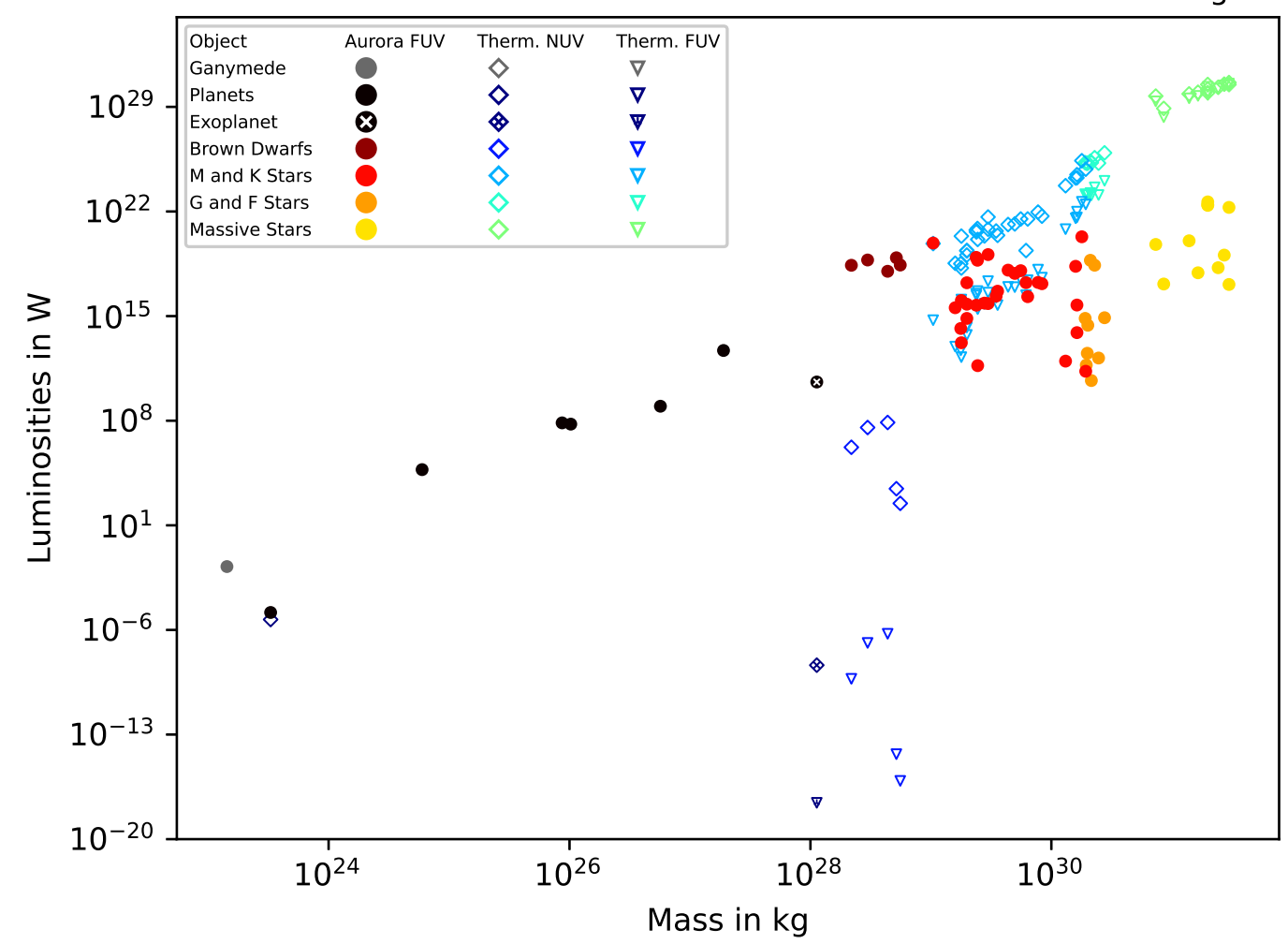

Fig. 6. Thermal UV luminosities for the NUV wavelength range (empty diamonds) and FUV wavelength range (empty triangles) compared to expected auroral FUV emission (full circles). For Brown dwarfs, the massive outer planets of the solar system, the exoplanet $\tau$ Boötis $\mathrm{b}$ and the moon Ganymede, the thermal UV emission is negligibly small compared to the possible auroral UV emission.

become a competing flux. G- and F-type stars possess thermal UV emission typically in excess of possibly expected UV auroral emission. This implies that auroral effects on a solar-type star will not be detectable within the NUV and FUV bands. However, if a significant fraction of the electromagnetic energy fluxes caused by the coupling of a companion is concentrated in line emission from its star, it might still be possible. Such observations do indeed exist. For example, Shkolnik et al. (2005, 2008) observe an excess in Ca II emission on HD 179949, $\tau$ Boötis, and $v$ Andromedae in synchrony with the orbital period of their close-in planetary companions. The energy fluxes associated with the Ca II surplus is estimated to be on the order of $\sim 10^{20} \mathrm{~W}$. This phenomenon is referred to as star-planet interaction (SPI) (e.g., Lanza 2008; Strugarek et al. 2015; Fischer \& Saur 2019). The energy flux models for the various scenarios in this work are applicable to the SPI studies. For massive stars, possible auroral UV luminosities are about 10 orders of magnitude lower than their thermal UV luminosities, and thus are expected to be undetectable. We note that the description of the thermal emission from one layer with a single effective temperature is a first-order approximation only, but should still provide a reasonable estimate for the detectability of auroral UV emission from outside the Solar System against the object's thermal UV emission.

\section{HST observations of brown dwarf 2MASS $1237+6526$}

After providing a basic framework for possible auroral emission from brown dwarfs in Sect. 2, we now analyze HST observations of the brown dwarf 2MASS J1237+6526 (Burgasser et al. 1999). The dwarf was observed with HST/STIS in 2020 during two HST visits, with two orbits in visit 1 and three orbits in visit 2, in order to search for UV auroral emission. The exposure details of these observations are given in Table 2. One orbit was dedicated to observation at NUV wavelengths, while Ly- $\alpha$ and FUV wavelengths were equally split up within four orbits in order to search for time variations in the aurora.

The results of our analysis are presented for each of the three wavelengths ranges in the next three subsections. The detailed steps of the data analysis are similar to those of the ultracool dwarf LSR J1835+3259 presented in Saur et al. (2018a) and described in Appendix B.1. The target is extremely faint in the UV and cannot be located on the detector by simple visual inspection. Therefore, we worked with the reference positions provided in the header of the data files (see Appendix B.1). Details of the acquisition of the faint target are provided in Appendix B.2. The error analysis and the associated calculation of the signal-to-noise $(\mathrm{S} / \mathrm{N})$ is based on the variance within the background fluxes in the $\mathrm{x} 2 \mathrm{~d}$-files, which provide the data at the highest calibration level (Appendix B.1). The resultant standard deviation $\sigma$ is then compared with the extracted signal from the target. We chose to work with the variance in the background because it covers nonsystematic, nonphysical contributions in the background, due to the standard processing pipeline performed by STScI, which can lead to large trends in the background fluxes or artificially negative values (see, e.g., Saur et al. 2018a or the STIS instrument handbook Riley et al. 2017). The error analysis is performed in parallel based on the counts in the flt-files, which provides the data at a lower calibration level (Appendix B.1). The two methods are not expected to give identical results due to the processing steps of the STScI pipeline converting flt to $\mathrm{x} 2 \mathrm{~d}$ files, for example due to the corrections in remapping resolutions elements between the flt and $\mathrm{x} 2 \mathrm{~d}$ data. The variance method also underestimates the statistical uncertainty in the case of large background fluxes as the method 
Table 2. Exposure details of HST/STIS observations of brown dwarf 2MASSS J1237+6526 (ID: 15870).

\begin{tabular}{|c|c|c|c|c|c|c|c|c|c|}
\hline Visit & Orbit & Exp & Root name & UT obs. date & UT obs. time ${ }^{(a)}$ & Exp. time & Type & Grating & Disp. \\
\hline$\#$ & \# & $\#$ & & yyyy-mm-dd & hh-mm-ss & $\mathrm{s}$ & & & $\AA \mathrm{pxl}^{-1}$ \\
\hline 1 & 1 & 1 & oe1g01010 & $2020-01-16$ & $19: 54: 07$ & 814 & Ly- $\alpha$ & G140M & 0.053 \\
\hline 1 & 1 & 2 & oe1g01020 & $2020-01-16$ & 20:11:09 & 807 & Ly- $\alpha$ & G140M & 0.053 \\
\hline 1 & 2 & 3 & oe1g01030 & $2020-01-16$ & $20: 32: 52$ & 1475 & NUV & G230L & 1.584 \\
\hline 1 & 2 & 4 & oe1g01040 & 2020-03-10 & $21: 24: 19$ & 1475 & NUV & G230L & 1.584 \\
\hline 2 & 1 & 5 & oe1g02010 & $2020-03-10$ & 09:37:12 & 872 & FUV & G140L & 0.584 \\
\hline 2 & 1 & 6 & oe1g02020 & $2020-03-10$ & $09: 55: 10$ & 871 & FUV & G140L & 0.584 \\
\hline 2 & 2 & 7 & oelg02030 & $2020-03-10$ & 10:13:07 & 1404 & FUV & G140L & 0.584 \\
\hline 2 & 2 & 8 & oe1g02040 & $2020-03-10$ & $11: 12: 39$ & 1055 & Ly- $\alpha$ & G140M & 0.053 \\
\hline 2 & 3 & 9 & oelg02050 & $2020-03-10$ & 11:38:30 & 1404 & FUV & G140L & 0.584 \\
\hline 2 & 3 & 10 & oe1g02060 & $2020-03-10$ & $12: 48: 04$ & 1159 & Ly- $\alpha$ & G140M & 0.053 \\
\hline
\end{tabular}

Notes. ${ }^{(a)}$ At the beginning of the exposures.

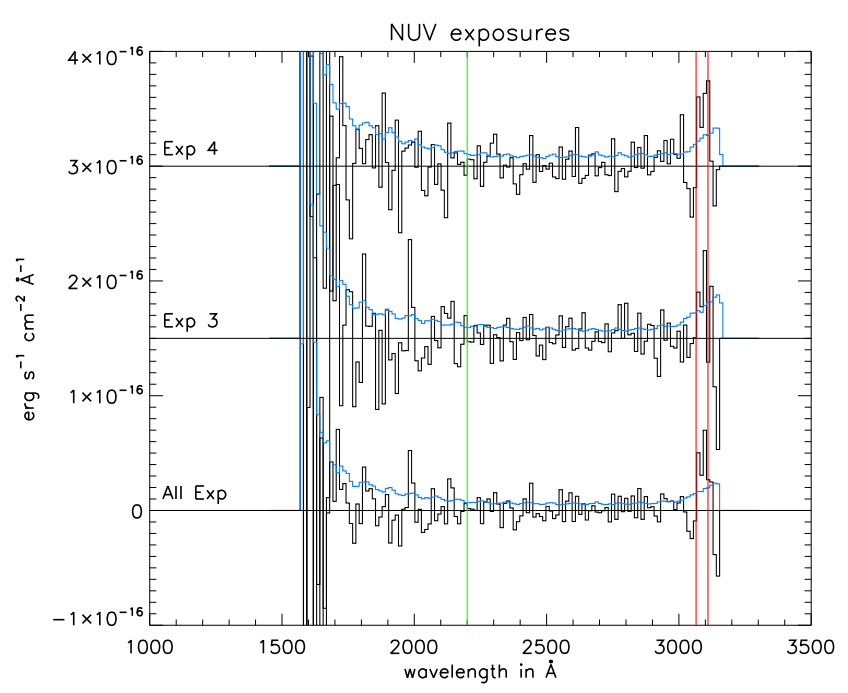

Fig. 7. Near-UV spectra of exposures 3 and 4, and combined spectrum. The spectra are separated by constant offsets for display purposes. The two red vertical lines indicate the wavelength band 3065-3110 A displaying the most significant flux within the spectra, which might stem from TiO emission. The green line indicates $2200 \AA$ (see text). In light blue we display the uncertainty spectrum calculated with the variance method described in Appendix B.1.

does not take into consideration the statistical uncertainty of the variance. Therefore it is worthwhile to provide $\mathrm{S} / \mathrm{N}$ values from both methods.

\subsection{Near-UV spectrum}

The NUV observations were executed with STIS grating G230L for the wavelength range 1570--3180 $\AA$. The resultant spectra obtained from the two exposures and the combined spectrum are shown in Fig. 7 as black lines. The light blue lines show the uncertainty spectra based on the variance of the background fluxes (Appendix B.1). At the lower end and somewhat at the higher end of the wavelength range the spectrum is noisy, similar to other HST/STIS observations with G230L resulting from the decreased sensitivity (see STIS Instrument Handbook).

The signal in the NUV is generally very faint and barely exceeds the noise level. Integrating between 2200 and $3000 \AA$, we find a flux of $1.6 \times 10^{-15} \mathrm{erg} \mathrm{s}^{-1} \mathrm{~cm}^{-2}$ with an $\mathrm{S} / \mathrm{N}$ of 2.4 .

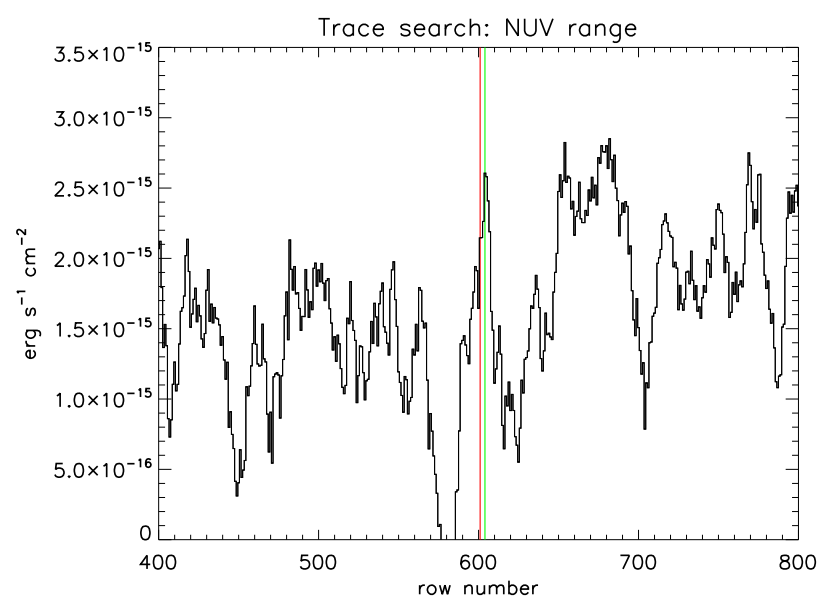

Fig. 8. Trace search for NUV signal. Integrated fluxes along the axis of dispersion (along rows) as a function of row number. The black curves shows integrated fluxes within the wavelength range 2200-3110 $\AA$ and the blue curve fluxes within 3065 and $3110 \AA$. The vertical red line indicates the row where the target is expected to be located and the green line indicates a shift of three rows where flux displays a local maximum.

Only within $3065-3110 \AA$ is a distinct feature visible (within the two red lines in Fig. 7). It possesses a net flux of $2.0 \times$ $10^{-15} \mathrm{erg} \mathrm{s}^{-1} \mathrm{~cm}^{-2}$ with an $\mathrm{S} / \mathrm{N}$ of 4.8 . We note, however, that the emission occurs near the long wavelength end of the grating G230L where the spectrum appears very noisy. Based on the counts of the signal and the background counts in the flt-files, we find an S/N of 3.8 within $3065-3110 \AA$. The counts over the lower wavelength range within 2200 and $3000 \AA$ are likely not significant because the net counts and resulting $\mathrm{S} / \mathrm{N}$ can have values higher or lower than one, depending on the details of choosing the rows to obtain the net signal and the rows for determining the background.

To further investigate the significance of the NUV signal from the target, we search for a signal by integrating along the axis of dispersion for each row within the unprocessed $\mathrm{x} 2 \mathrm{~d}$ files (i.e., without subtracting background emission). The results are shown in Fig. 8 for integration within the wavelength range 3065 and $3110 \AA$. The vertical red line in Fig. 8 shows the row where the target is expected to be located, and the green line is shifted by three rows to better fit the local maximum of the 


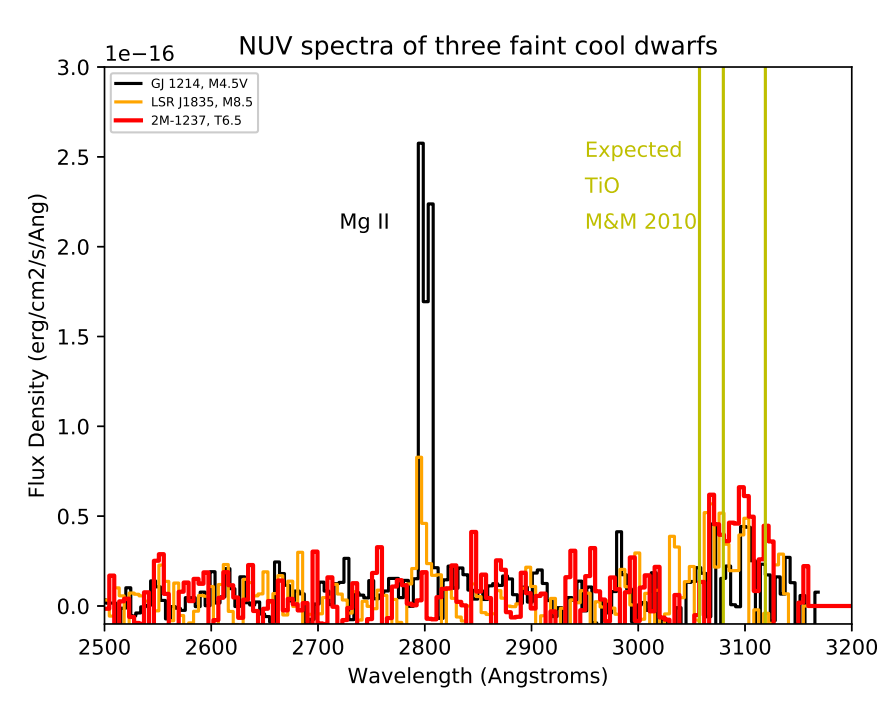

Fig. 9. Near-UV spectrum of 2MASS J1237+6526 compared with the M8.5 ultracool dwarf LSR J1835+3259 and M4.5 low mass star GJ 1214. Around $2800 \AA$, the Mg II doublet is visible. The vertical yellow lines show TiO bands based on Miliordos \& Mavridis (2010).

flux. This small shift lies within the pointing uncertainties. A localized enhancement of integrated flux within 3065-3110 is visible near the row where the target is expected to be located. Its amplitude is consistent with the derived flux of $1.6 \times$ $10^{-15} \mathrm{erg} \mathrm{s}^{-1} \mathrm{~cm}^{-2}$. However, Fig. 8 also shows that the background is highly inhomogeneous with other peaks of similar amplitudes. This cautions against a claim of an unambiguous detection of a NUV signal from the source.

The origin of the possible net emission within $3065-3110 \AA$ is not clear, but it could stem from emission of TiO possibly excited by auroral electrons (Palmer \& Hsu 1972; Phatak \& Palmer 1970; Miliordos \& Mavridis 2010). Low S/N emission in the same wavelength range was also observed in the spectra of the ultracool M8.5 dwarf LSR J1835+3259 (Saur et al. 2018a). The same spectral region is displayed in more detail in Fig. 9, where we also show three expected $\mathrm{TiO}$ vibrational bands (yellow vertical lines) at 3057.4, 3079.7, and 3119.1 $\AA$ calculated based on data in Miliordos \& Mavridis (2010). While the lower two wavelength bands only approximately coincide with peaks in the observation, the band at $3119.1 \AA$ fits closer to an observed peak (red line). The wavelengths of the lower bands, however, are also less certain compared to the band at $3119.1 \AA$ (Miliordos \& Mavridis 2010). For example, the band at $3079.7 \AA$ originates from the ${ }^{\prime} v=2$ level of $\mathrm{G}^{3} \mathrm{H}$ state which would require a favorable Franck-Condon factor for excitation. We note that $\mathrm{TiO}$ emission from the upper atmosphere of a $\mathrm{T}$ dwarf would be surprising since $\mathrm{TiO}$ is expected to be depleted in the L-dwarf stage and rained out as minerals (e.g., Burrows et al. 2000; Reid 2013).

All in all, the analysis shows that the low $\mathrm{S} / \mathrm{N}$ feature between 3065-3110 ̊ within the inhomogeneous background flux needs to be taken with caution. Keeping this uncertainty in mind, we compare the NUV spectrum of the T-type brown dwarf 2MASS J1237+6526 with slightly more massive dwarfs, such as the ultracool M8.5 dwarf LSR J1835+3259 (Saur et al. 2018a) and the M4.5V star GJ 1214 (France et al. 2016). The spectra of these three targets are displayed in Fig. 9. Fluxes associated with Mg II at 2796 and $2803 \AA$ (e.g., Feldman et al. 1996; France

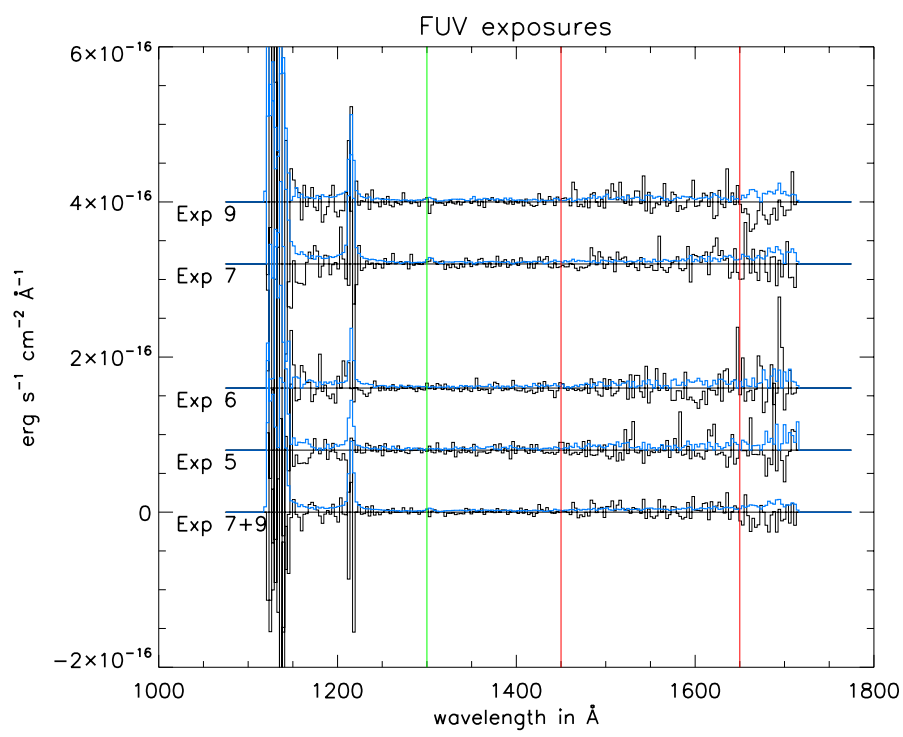

Fig. 10. Far-UV spectra of 2 MASS J1237+6526. The spectra are shifted by a constant offset for display purposes. The red lines at 1450 and $1650 \AA$ and the green line at $1300 \AA$ indicate the region within which integrated fluxes are calculated (see text). In light blue we display the uncertainty spectrum calculated with the variance method described in Appendix B.1.

et al. 2013) are not detectable within the spectrum of 2MASS $\mathrm{J} 1237+6526$, but grow with increasing mass of the targets. The trend of increasing Mg II luminosity with mass holds on average also for more massive $\mathrm{M}$ and $\mathrm{K}$ dwarfs observed within the MUSCLES program by France et al. (2013, 2016). The tentative emission possibly stemming from TiO within 3065 and $3110 \AA$ is largest for 2MASS J1237+6526, which is the coldest and lowest mass object of the three. The flux in the same band is lower for LSR J1835+3259, while it appears negligible for GJ 1214.

\subsection{Far-UV spectrum}

The FUV observations were taken with STIS grating G140L within the wavelength range 1150-1730 $\AA$ (see Table 2). The results of each exposure and exposures 7 and 9 combined are shown in Fig. 10. The light blue lines show the residual spectra based on the variance method (Appendix B.1).

The brown dwarf is overall extremely faint in the FUV. The only wavelength range with somewhat enhanced fluxes lies within 1450 and $1650 \AA$. Between 1300 and $1450 \AA$ the fluxes are negligible. For wavelengths shorter than $1300 \AA$ and longer than $1650 \AA$ the sensitivity is low compared to the mid-wavelength range and the resultant spectrum turns noisy without significant flux consistent with the uncertainty spectrum in Fig. 10. The flux within 1450 and $1650 \AA$ differs between the four exposures. It is not significant for exposures 5 and 6 taken during visit 2 with an $S / N<1$. Exposures 7 and 9 taken during visit 2 lead to an integrated flux of $4.0 \times 10^{-16} \mathrm{erg} \mathrm{s}^{-1} \mathrm{~cm}^{-2}$ with an $\mathrm{S} / \mathrm{N}$ of 2.6 and $7.8 \times 10^{-16} \mathrm{erg} \mathrm{s}^{-1} \mathrm{~cm}^{-2}$ with an S/N of 4.6, respectively. An S/N estimate based on counts in the flt-flies within 1450 and $1650 \AA$ (see Appendix B.1) for exposure 9 leads to an $\mathrm{S} / \mathrm{N}$ of 3.5.

To independently assess the significance of the fluxes from the target for exposures 7 and 9 combined, we integrate the fluxes within each row (i.e., along the direction of dispersion) within 1450 and $1650 \AA$ for each row. These integrated fluxes are displayed as a function of row number in Fig. 11. The vertical red 


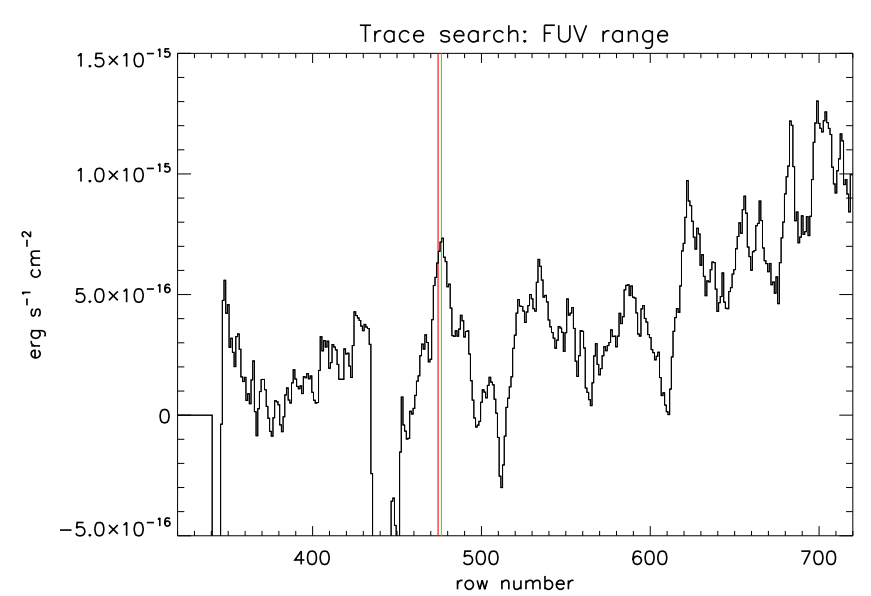

Fig. 11. Trace search at FUV wavelength for 2 MASS J1237+6526. Integrated fluxes along rows within 1450 and $1650 \AA$ (i.e., along the axis of dispersion), displayed as a function of row number (exposures 7 and 9 combined). The vertical red line indicates the row where the target is expected to be located, and the green line indicates a shift of two rows where the flux displays a local maximum.

line indicates the row where the target is expected to be located, which coincides with a local maximum of the observed flux. The green line is shifted by two rows, which slightly fits better and which is used as target location for calculating the fluxes in this subsection. This small shift lies within the pointing uncertainties. Figure 11 shows both a wide variability and a trend in the background fluxes preventing a unique detection.

Possible emission between 1450 and $1650 \AA$ would be consistent with expectations from $\mathrm{H}_{2}$ Lyman and Werner bands as observed, for example, with the Galileo Ultraviolet Spectrometer and the Hopkins Ultraviolet Telescope (Pryor et al. 1998; Wolven \& Feldman 1998). In particular, peak fluxes from 2MASS J1237+6526 at wavelength bands slightly longer than $1600 \AA$ are also observed and theoretically expected from Jupiter's auroral emission (Wolven \& Feldman 1998). Expected smaller amplitude features for lower FUV wavelengths from $\mathrm{H}_{2}$ Lyman and Werner bands might be too weak to be resolved in the dwarf spectrum. The possible $\mathrm{H}_{2}$ emission cannot stem from the geocorona because of the extremely low $\mathrm{H}_{2}$ number densities at and above HST altitudes due to the rapid oxidation of $\mathrm{H}_{2}$ and conversion to $\mathrm{H}$ atoms in the Earth's upper atmosphere below $200 \mathrm{~km}$. This is consistent with all previous analyses of HST observations at these wavelengths.

\section{3. $L y-\alpha$ spectrum}

Lyman- $\alpha$ emission is searched for with STIS grating G140M at central wavelength $1218 \AA$. In Figs. 12 and 13, we display the Ly- $\alpha$ spectrum for visit 1 and visit 2, respectively. The top panels in both figures show the total Ly- $\alpha$ fluxes in black for rows where the target is located. The averaged Ly- $\alpha$ flux in rows away from the target is shown in green. These fluxes result from the geocoronal emission, and need to be subtracted to retain the net fluxes from the target. These net fluxes are displayed in the lower panels of Figs. 12 and 13 together with the uncertainty spectrum in light blue based on the variance method in the Appendix. We note, in the case of larger background fluxes such as geocoronal emission, that it underestimates the uncertainty, and that this effect leads to lower $\mathrm{S} / \mathrm{N}$ values as those based on counts (see discussion below). Therefore larger negative outliers of the net

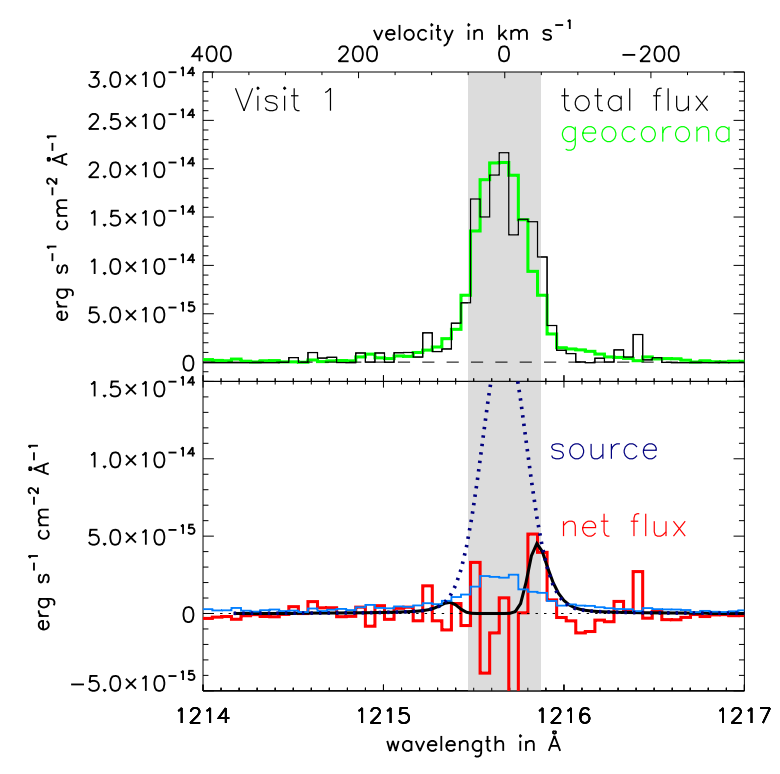

Fig. 12. Ly- $\alpha$ spectrum of Visit 1 of 2MASS J1237+6526. Top panel: total flux and geocoronal emission within the slit determined from rows away from the target. Bottom panel: net flux from the target and the possible source flux (see text for details). In light blue we display the uncertainty spectrum calculated with the variance method described in Appendix B.1.

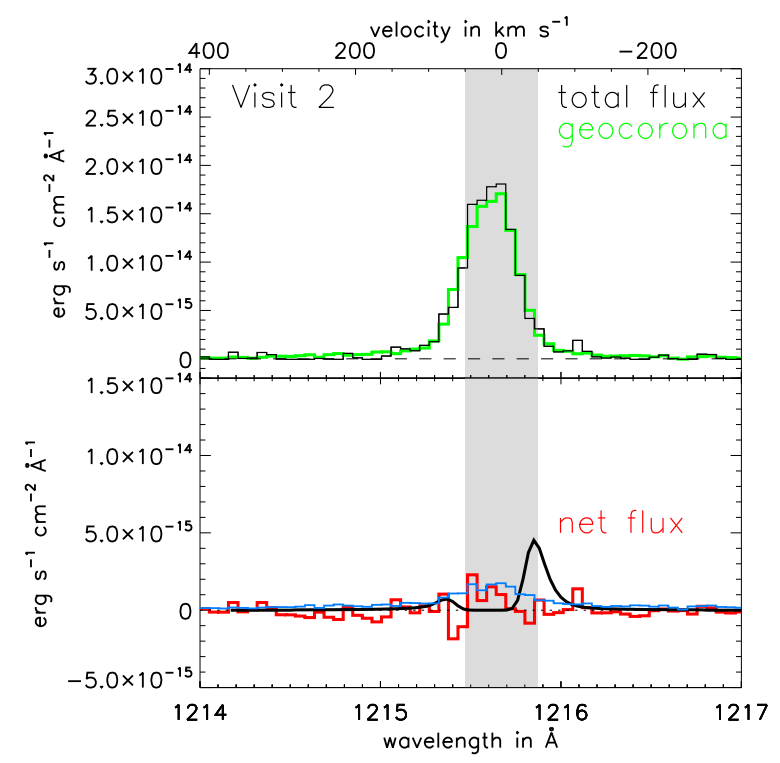

Fig. 13. Ly- $\alpha$ spectrum of Visit 2 of 2MASS J1237+6526. Labels are the same as in Fig. 12.

flux are possible at wavelengths with large geocoronal emission (displayed as a green line). Due to absorption in the interstellar medium, emission near the central Ly- $\alpha$ wavelength $1215.67 \AA$ is not detectable (Linsky \& Wood 1996, 2014; France et al. 2013). The spectral range where the geocorona is dominant is shown as a gray shaded area in both figures. The net emission observed for visit 1 shows a small surplus in the Ly- $\alpha$ red wing longward of $1215.8 \AA$. The net flux within the wavelength range 1215.8 to $1216.5 \AA$ is $4.9 \times 10^{-16} \mathrm{erg} \mathrm{s}^{-1} \mathrm{~cm}^{-2}$ with an $\mathrm{S} / \mathrm{N}$ of 3.7 based on the variance method described in the Appendix. In contrast, visit 2 displayed in Fig. 13, shows no sign of a surplus in either the red or the blue wings. Due to large background counts 
Table 3. Possible values and upper limits for the UV luminosities of 2MASS J1237+6526 (see text).

\begin{tabular}{lcrrr}
\hline \hline Type & $\begin{array}{c}\text { Wavelength band } \\
\AA\end{array}$ & $\begin{array}{r}\text { Flux } \\
\mathrm{Am}^{-2}\end{array}$ & $\begin{array}{r}S / N^{(a)} \\
\mathrm{erg} \mathrm{s}^{-1} \mathrm{Cm}^{-15}\end{array}$ & $\begin{array}{r}\text { Luminosity } \\
\text { watt }\end{array}$ \\
\hline NUV exposures \#3+\#4 & $3065-3110$ & $1.6 \times 10^{-15}$ & 3.8 & $2.1 \times 10^{18}$ \\
FUV exposure \#9 & $1450-1650$ & $7.9 \times 10^{-16}$ & 3.5 & $1.0 \times 10^{18}$ \\
Ly- $\alpha$ exposures \#1+\#2 & $1215.8-1215.5$ & $6.2 \times 10^{-16}$ & 1.9 & $7.6 \times 10^{18}$ \\
\hline
\end{tabular}

Notes. ${ }^{(a)}$ Lowest value of $\mathrm{S} / \mathrm{N}$ from the methods based on variances or counts (see main text).

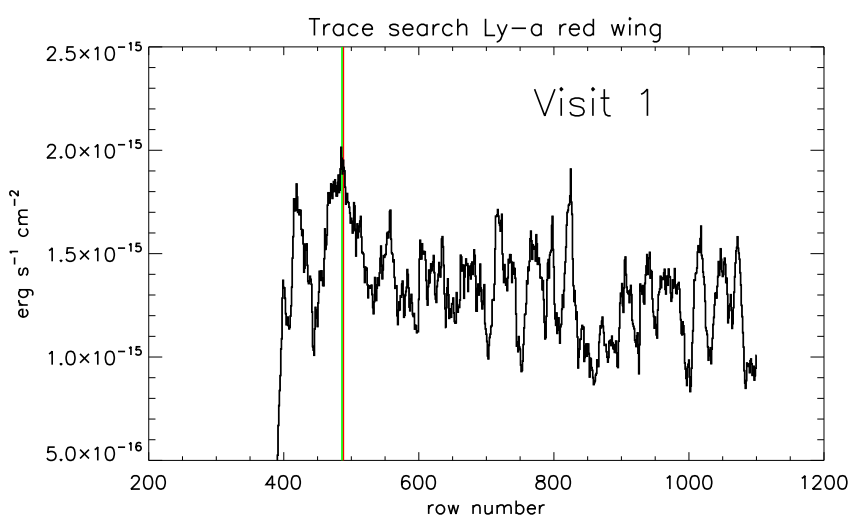

Fig. 14. Trace search at Ly- $\alpha$ redwing for 2 MASS J1237+6526.

from the geocorona, the $\mathrm{S} / \mathrm{N}$ calculations based on the variance methods overestimate the $\mathrm{S} / \mathrm{N}$. The large background flux is considered in the $\mathrm{S} / \mathrm{N}$ calculations based on the counts in the flt-files. This therefore leads to a lower $\mathrm{S} / \mathrm{N}$ of 1.9 . We note that we chose to subtract the background from the trace flux within the $\mathrm{x} 2 \mathrm{~d}-\mathrm{files}$ because small deviations in the not-fully-rectified fltfiles lead to spatial uncertainties in the background count structure, which can introduce significant errors in the subtraction of these files.

The possible surplus in the red wing of visit 1 is further investigated in Fig. 14, where we integrate the flux (without any background subtraction) in each row between 1215.8 and $1216.5 \AA$ and show this flux as a function of row number. The row where the target is expected to be located is shown as red vertical line in Fig. 14. It is colocated with the maximum flux demonstrating independently a small possible net surplus within the Ly- $\alpha$ red wing. The local maximum appears to be slightly shifted 2 rows downward (green line in the figure). Such a small offset is typical within the pointing uncertainties. Figure 14 shows that the background emission possesses wide variability including a few other large peaks of nearly similar height, which introduces uncertainty in the unique detection of a signal from the source.

The possible net flux in Fig. 12 can be used to roughly estimate the underlying Ly- $\alpha$ source emission. We apply the same procedure as in Saur et al. (2018a) based on reconstruction techniques from Woods et al. (2005), France et al. (2013), Bourrier et al. (2015), and Youngblood et al. (2016), among others. Since the observed emission is very noisy we refrain from applying a quantitative inversion to obtain the source function and unconstrained parameter of the interstellar medium. Instead we manually explore parameter space and present a reasonable fit with the aim of having an order of magnitude constraint of the initial source luminosity. Therefore, the source spectrum is modeled with a Voigt profile with a Doppler width of
$40 \mathrm{~km} \mathrm{~s}^{-1}$, a damping parameter of 0.09 , and a peak flux of $2 \times$ $10^{-14}$ erg $\mathrm{s}^{-1} \mathrm{~cm}^{-2} \AA^{-1}$. The absorption in the interstellar medium by hydrogen $\mathrm{H}$ I and deuterium $\mathrm{D}$ I is described using a Lorentzian absorption with cross-sections and ratios given in Saur et al. (2018a). We assume a hydrogen column density of the interstellar medium of $1 \times 10^{17} \mathrm{~cm}^{-2}$ consistent with estimates from the LISM calculator (Redfield \& Linsky 2008). The radial velocity of the brown dwarf 2MASS J1237+6526 is unknown and the velocities of the interstellar medium are only a few $\mathrm{km} \mathrm{s}^{-1}$ based on the LISM kinetic calculator (Redfield \& Linsky 2008). For a reasonable fit to the data we assume a relative velocity between the source of the emission and the ISM of $16 \mathrm{~km} \mathrm{~s}^{-1}$. However, velocities of $8 \mathrm{~km} \mathrm{~s}^{-1}$ and a column density of $2 \times 10^{16}$ $\mathrm{cm}^{-2}$ or a velocity of $10 \mathrm{~km} \mathrm{~s}^{-1}$ or $1 \times 10^{16} \mathrm{~cm}^{-2}$ give equally good fits. The resultant Ly- $\alpha$ profile is convolved with the line spread function from the STIS Instrument Handbook (Riley et al. 2017). In Fig. 12 we show the resultant fit by eye as black curve. The underlying Ly- $\alpha$ source spectrum from the brown dwarf 2MASS J1237+6526 is displayed as a dashed line in the figure. Integrating the source Ly- $\alpha$ flux derived for visit 1 leads to a total flux of $5.8 \times 10^{-15} \mathrm{erg} \mathrm{s}^{-1} \mathrm{~cm}^{-2}$.

Only for guiding the eye, we display in Fig. 13 the fit to the net flux from visit 1 (black line). This demonstrates that no net Ly- $\alpha$ was detected during visit 2 in excess of the noise level.

\subsection{Band luminosities of target}

With the possible fluxes derived in the previous subsections, we calculate possible luminosities for the associated wavelength bands. They are summarized in Table 3 and displayed in Fig. 15 as red triangles. The formal $\mathrm{S} / \mathrm{N}$ in the observations are shown with the red vertical bars around the triangle. As the derived values are subject to additional uncertainty due to the background subtraction which introduces systematic uncertainties that are difficult to fully assess, the derived luminosities need to be considered with appropriate caution, but can be considered upper limits in any case. We indicate this symbolically with the thin downward arrows. Luminosities at $\mathrm{H}-\alpha$ and radio wavelengths are taken from the literature (Burgasser et al. 2002; Kao et al. 2018). For comparison we show expected auroral emission with the scaling model derived in Sect. 2. The orange circles show the expected auroral luminosities based on expression (9) with the auroral luminosities from Jupiter $L_{\text {mag,J }}$, the auroral power potential $S_{\text {pot,rel }}$ of 2 MASS J1237+6526 relative to Jupiter and assuming $Q_{\text {rel }}=1$ (i.e., that the auroral generator within the magnetosphere of 2MASS J1237+6526 is similar to that of Jupiter).

\section{Discussion and conclusion}

In this section we discuss further under what circumstances the possible UV emission from 2MASS J1237+6526 could originate from auroral processes by using the expressions for 


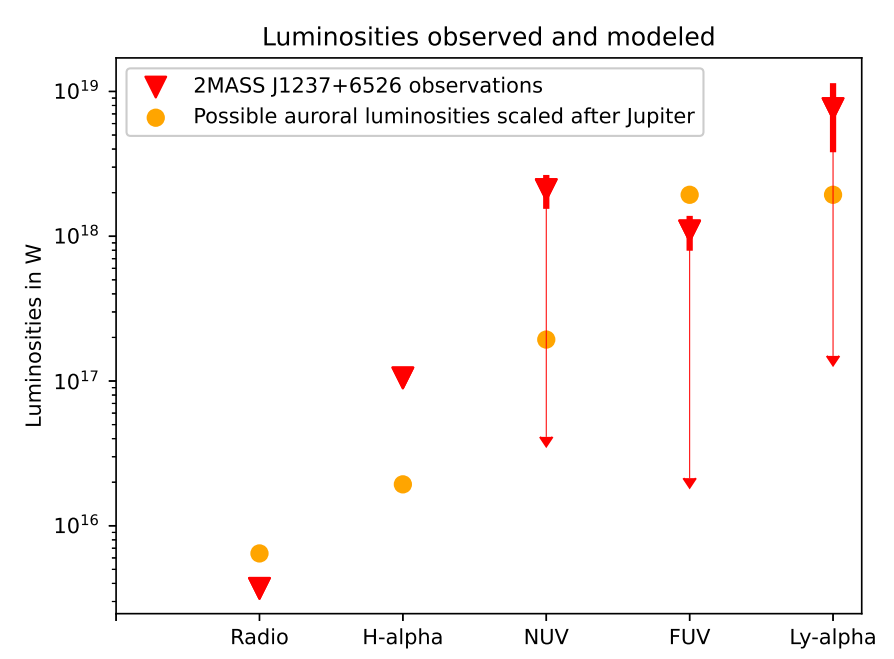

Fig. 15. Possible luminosities of brown dwarf 2MASS J1237+6526 (red triangles) compared with theoretically derived auroral luminosities (orange circles). Auroral luminosities are scaled after Jupiter and are calculated with $L_{\text {Jupiter }} S_{\text {pot,rel }} Q_{\text {rel }}(=1)$ (see text for details). The derived luminosities need to be considered with caution, but can be considered upper limits in any case, which is indicated symbolically as downward arrows.

auroral powers from Sect. 2.3 for a mass flow driven or companion driven aurora, respectively. Our analysis of HST observations shows that the derived UV luminosities of 2MASS $\mathrm{J} 1237+6526$, if confirmed, lie in the range $10^{18}$ to $10^{19}$ watt in the NUV, FUV, and at Ly- $\alpha$ wavelengths. They otherwise provide upper limits. Jupiter's total auroral UV emission $P_{\text {Jupiter }}$ lies in the range $2-10 \times 10^{12}$ watt and is thus a factor of $\sim 10^{6}$ smaller. Based on the derivations in Sect. 2, the auroral power potential of Jupiter is $S_{\text {pot,J }}=600$ watt $\mathrm{m}^{-2}$ Siemens $^{-1}$ and the power potential of 2 MASS J1237 +6526 is $S_{\text {pot,2M }}=3.9 \times$ $10^{8}$ watt $\mathrm{m}^{-2}$ Siemens $^{-1}$. This leads to a relative power potential of $S_{\text {pot,rel }}=S_{\text {pot }, 2 \mathrm{M}} / S_{\text {pot, }}=6.5 \times 10^{5}$. Thus, the relative power potential differences could explain the large difference between Jupiter's UV emission and the possible UV emission from 2MASS J1237+6526. This is also shown in Fig. 15, where we use the average values of the UV luminosities for Jupiter from Table 1 combined with the scaling model from expression (9). The comparison shows that within an order of magnitude the auroral luminosities and possible UV emission from 2MASS J1237+6529 could be comparable.

Alternatively, a planetary companion can explain the possible UV emission as well. This scenario is described by expression (15). The total UV luminosity of Io's northern and southern auroral spots $P_{\mathrm{Io}}$ combined lies in the range of $1-10 \times 10^{10}$ watt (Prangé et al. 1996; Wannawichian et al. 2010; Bonfond et al. 2013), about a factor of 100 smaller compared to Jupiter's main auroral emission driven by mass transport. The same power potential is applicable for both types of aurora, which means that the luminosity in (15) can render values comparable to the observation with $Q_{\text {rel }}=100$. This is possible, for example, for a planetary companion at the same radial distance as Io from Jupiter $\left(5.9 R_{\mathrm{J}}\right)$, but with a radius 10 times larger than Io's $\left(R_{\mathrm{Io}}=1800 \mathrm{~km}\right)$, corresponding to 2.8 times the radius of Earth, or, alternatively, an Earth-sized planet at a distance of $3.5 R_{\mathrm{J}}$. Earth-sized planets around brown dwarfs are thought to be possible (Payne \& Lodato 2007), but no short-period companion around 2MASS J1237+6526 has been reported as transits in photometric surveys to the authors' knowledge.
Both auroral scenarios would be consistent with the observed H- $\alpha$ emission from the dwarf (Burgasser et al. 2002; Liebert $\&$ Burgasser 2007). They could also be the driver of the radio emission reported in Kao et al. (2016, 2018).

Due to the higher mass of 2MASS J1237+6526 compared to that of Jupiter by a factor of about 30, the orbital period of a companion located at a distance similar to Jupiter's moon Io would be shorter by a factor of 5.5 with a rotation period of $7.8 \mathrm{hr}$. Depending on the location where the coupling of a planetary companion intersects the surface of the brown dwarf, Doppler shifts of the emission due to the rotation of the dwarf on the order of $10 \mathrm{~km} \mathrm{~s}^{-1}$ could emerge. This would contribute to the Doppler shift of the Ly- $\alpha$ emission. It might also allow the source to be identified by observations of periodic oscillations of the shift in future measurements.

Any variability of the possible auroral UV emission from the brown dwarf during the different FUV and Ly- $\alpha$ visits could in principle be explained by a planetary companion whose auroral effects are obstructed (or partially obstructed) because they lie behind the dwarf as seen from Earth (see discussion in e.g. Fischer \& Saur 2019). However, tilted magnetic fields of the central body can cause time variability as well. Due to the uncertainty of the significance of the HST observations, it is premature to explore the various possibilities further. We note that $\mathrm{H}-\alpha$ emission was observed to be persistent within a factor of two (Burgasser et al. 2002).

An important question is whether the $\mathrm{H}-\alpha$ emission and the possible UV emission from 2MASS J1237+6526 could be caused by chromospheric emission. Liebert \& Burgasser (2007) argue that this brown dwarf does not possess a very active chromosphere. One of their arguments is based on the very faint absolute $J$-band magnitude of $15.88 \pm 0.13$ (Vrba et al. 2004), which is similar to other T6-7 dwarfs and rules out that 2MASS J1237+6526 is overluminous. In addition, the kinematics of the objects measured by Vrba et al. (2004) suggest that it is not a young object. Finally, its near-IR spectrum provides evidence that 2MASS J1237+6526 has a high surface gravity and/or a low metallicity, which implies that the dwarf is old (Liebert \& Burgasser 2007). These arguments speak against an overluminous chromosphere as the source of the $\mathrm{H}-\alpha$ and UV emission. However, chromospheric (i.e., internally driven) activity is not very well understood for L- and T-type brown dwarfs. Therefore, it remains an open question whether observed emission at $\mathrm{H}-\alpha$, radio, or possibly UV wavelengths from 2MASS $\mathrm{J} 1237+6526$ is externally driven by auroral acceleration process or by internal heating due to Alfvén waves, for example (e.g., Rodríguez-Barrera et al. 2018).

In summary, the possible UV emission of the brown dwarf 2MASS J1237+6526 are consistent with theoretically expected auroral emission driven in the dwarf's magnetosphere by mass transport or a planet-sized companion. However, the observational results of this analysis are tentative and need to be considered with caution due to the low $\mathrm{S} / \mathrm{N}$ in combination with the nonsystematic background variations. We also did not detect uniquely identifiable spectral lines and bands in the NUV and FUV spectrum (except possibly Ly- $\alpha$ ). Further observations are required to establish the significance of the UV emission. Resolving time variability could constrain the auroral generator, and thus it will be possible to determine whether the emission is due to an external source, due to auroral particle acceleration, in contrast to internally produced plasma heating including internally driven electron beams. Despite the tentativeness of the presented observations, brown dwarfs are ideal objects to search for UV aurora outside of the Solar System, and further UV 
observations might prove greatly helpful in understanding the space plasma environment around brown dwarfs and extrasolar planets.

Acknowledgements. This work is based on observations with the NASA/ESA Hubble Space Telescope obtained at the Space Telescope Science Institute which is operated by the Association of Universities for Research in Astronomy (AURA), Inc., under NASA contract NAS 5-26555. We highly appreciate the help of Michael Leveille in scheduling the observations and the valuable comments of Johns Debes and his team on the data analysis. This project has received funding from the European Research Council (ERC) under the European Union's Horizon 2020 research and innovation programme (grant agreement No. 884711). DFS was supported by NASA through Grant HST-GO15870.002-A from the Space Telescope Science Institute. This research has made use of the SIMBAD database, operated at CDS, Strasbourg, France. This work has made use of data from the European Space Agency (ESA) mission Gaia (https: //www. cosmos.esa.int/gaia), processed by the Gaia Data Processing and Analysis Consortium (DPAC, https://www. cosmos.esa.int/web/ gaia/dpac/consortium). Funding for the DPAC has been provided by national institutions, in particular the institutions participating in the Gaia Multilateral Agreement.

\section{References}

Anderson, B. J., Johnson, C. L., Korth, H., et al. 2011, Science, 333, 1859 Artigau, E., Nadeau, D., \& Doyon, R. 2003, IAU Symp., 211, 451

Baines, E. K., \& Armstrong, J. T. 2012, ApJ, 744, 138

Banaszkiewicz, M., Axford, W. I., \& McKenzie, J. F. 1998, A\&A, 337, 940

Barnes, J. R., Haswell, C. A., Jeffers, S. V., et al. 2016, in 19th Cambridge Workshop on Cool Stars, Stellar Systems, and the Sun (CS19) (Cambridge: Cambridge University Press), 132

Basri, G., \& Marcy, G. W. 1988, ApJ, 330, 274

Berdyugina, S. V., Harrington, D. M., Kuzmychov, O., et al. 2017, ApJ, 847, 61

Berger, E. 2006, ApJ, 648, 629

Berger, E., Basri, G., Gizis, J. E., et al. 2008, ApJ, 676, 1307

Bhardwaj, A., \& Gladstone, G. R. 2000, Rev. Geophys., 38, 295

Blackwell, D. E., \& Lynas-Gray, A. E. 1994, A\&A, 282, 899

Bonfanti, A., Ortolani, S., \& Nascimbeni, V. 2016, A\&A, 585, A5

Bonfond, B., Hess, S., Gérard, J.-C., et al. 2013, Planet. Space Sci., 88, 64

Boro Saikia, S., Jeffers, S. V., Morin, J., et al. 2016, A\&A, 594, A29

Borsa, F., Scandariato, G., Rainer, M., et al. 2015, A\&A, 578, A64

Bouchy, F., Udry, S., Mayor, M., et al. 2005, A\&A, 444, L15

Bourrier, V., Ehrenreich, D., \& Lecavelier des Etangs, A. 2015, A\&A, 582, A65

Boyajian, T., von Braun, K., Feiden, G. A., et al. 2015, MNRAS, 447, 846

Broadfoot, A. L., Belton, M. J. S., Takacs, P. Z., et al. 1979, Science, 204, 979

Brogi, M., Snellen, I. A. G., de Kok, R. J., et al. 2012, Nature, 486, 502

Burgasser, A. J., Kirkpatrick, J. D., Brown, M. E., et al. 1999, ApJ, 522, L65

Burgasser, A. J., Kirkpatrick, J. D., Reid, I. N., et al. 2000, AJ, 120, 473

Burgasser, A. J., Liebert, J., Kirkpatrick, J. D., \& Gizis, J. E. 2002, AJ, 123, 274

Burrows, A., Marley, M. S., \& Sharp, C. M. 2000, ApJ, 531, 438

Burrows, A., Hubbard, W. B., Lunine, J. I., \& Liebert, J. 2001, Rev. Mod. Phys., 73,719

Casagrande, L., Flynn, C., \& Bessell, M. 2008, MNRAS, 389, 585

Chabrier, G., \& Baraffe, I. 2000, ARA\&A, 38, 337

Clark, G., Tao, C., Mauk, B. H., et al. 2018, J. Geophys. Res. Space Phys., 123, 7554

Clem, J. L., VandenBerg, D. A., Grundahl, F., \& Bell, R. A. 2004, AJ, 127, 1227

Connerney, J. E. P., Baron, R., Satoh, T., \& Owen, T. 1993, Science, 262, 1035

Connerney, J. E. P., Kotsiaros, S., Oliversen, R. J., et al. 2018, Geophys. Res. Lett., 45, 2590

Delitsky, M. L., \& Lane, A. L. 1998, J. Geophys. Res., 103, 31391

Delrez, L., Gillon, M., Triaud, A. H. M. J., et al. 2018, MNRAS, 475, 3577

Dessler, A. J. 1980, Icarus, 44, 291

Donati, J. F. 1999, MNRAS, 302, 457

Donati, J. F., Moutou, C., Farès, R., et al. 2008, MNRAS, 385, 1179

Dougherty, M. K., Cao, H., Khurana, K. K., et al. 2018, Science, 362, aat5434

Eviatar, A., Strobel, D. F., Wolfven, B. C., et al. 2001, ApJ, 555, 1013

Feldman, P. D., Weaver, H. A., Boice, D. C., \& Stern, S. A. 1996, Icarus, 121, 442

Feldman, P. D., Most, H. W., Retherford, K., et al. 2000, Geophys. Res. Lett., 27, 1787

Filippazzo, J. C., Rice, E. L., Faherty, J., et al. 2015, ApJ, 810, 158

Fischer, C., \& Saur, J. 2019, ApJ, 872, 113

Fossati, L., Kochukhov, O., Jenkins, J. S., et al. 2013, A\&A, 551, A85

France, K., Froning, C. S., Linsky, J. L., et al. 2013, ApJ, 763, 149

France, K., Loyd, R. O. P., Youngblood, A., et al. 2016, ApJ, 820, 89
Fröhlich, H. E. 2007, Astron. Nachr., 328, 1037

Gaia Collaboration (Brown, A. G. A., et al.) 2018, A\&A, 616, A1

Gaidos, E., \& Mann, A. W. 2014, ApJ, 791, 54

Gaidos, E., Mann, A. W., Lépine, S., et al. 2014, MNRAS, 443, 2561

Gaulme, P., Vannier, M., Guillot, T., et al. 2010, A\&A, 518, L153

Gizis, J. E., Monet, D. G., Reid, I. N., et al. 2000, AJ, 120, 1085

Goertz, C. K. 1980, J. Geophys. Res., 85, 2949

Goldreich, P., \& Lynden-Bell, D. 1969, ApJ, 156, 59

Grieves, N., Ge, J., Thomas, N., et al. 2018, MNRAS, 481, 3244

Hall, D. T., Feldman, P. D., McGrath, M. A., \& Strobel, D. F. 1998, ApJ, 499, 475

Hallinan, G., Antonova, A., Doyle, J. G., et al. 2008, ApJ, 684, 644

Hallinan, G., Littlefair, S. P., Cotter, G., et al. 2015, Nature, 523, 568

Hatzes, A. P., \& Rauer, H. 2015, ApJ, 810, L25

Hayashi, C., \& Nakano, T. 1963, Prog. Theor. Phys., 30, 460

Helling, C., \& Rimmer, P. B. 2019, Phil. Trans. R. Soc. London Ser. A, 377, 20180398

Henry, G. W., \& Winn, J. N. 2008, AJ, 135, 68

Hill, T. W. 1979, J. Geophys. Res., 84, 6554

Hill, T. W. 2001, J. Geophys. Res., 106, 8101

Hohle, M. M., Neuhäuser, R., \& Schutz, B. F. 2010, Astron. Nachr., 331, 349

Hubrig, S., Briquet, M., De Cat, P., et al. 2009, Astron. Nachr., 330, 317

Jones, B. W. 2004, Life in the Solar System and Beyond (London: Springer)

Kao, M. M., Hallinan, G., Pineda, J. S., et al. 2016, ApJ, 818, 24

Kao, M. M., Hallinan, G., Pineda, J. S., Stevenson, D., \& Burgasser, A. 2018, ApJS, 237, 25

Kervella, P., Mérand, A., Ledoux, C., Demory, B. O., \& Le Bouquin, J. B. 2016, A\&A, 593, A127

Kim, Y.-C., \& Demarque, P. 1996, ApJ, 457, 340

Kirkpatrick, J. D. 2005, ARA\&A, 43, 195

Kivelson, M. G., Khurana, K. K., \& Volwerk, M. 2002, Icarus, 157, 507

Kovári, Z., Strassmeier, K. G., Granzer, T., et al. 2004, A\&A, 417, 1047

Kumar, S. S. 1962, AJ, 67, 579

Lanza, A. F. 2008, A\&A, 487, 1163

Lépine, S., Rich, R. M., \& Shara, M. M. 2003, AJ, 125, 1598

Lépine, S., Hilton, E. J., Mann, A. W., et al. 2013, AJ, 145, 102

Liebert, J., \& Burgasser, A. J. 2007, ApJ, 655, 522

Linsky, J. L., \& Wood, B. E. 1996, ApJ, 463, 254

Linsky, J. L., \& Wood, B. E. 2014, ASTRA Proc., 1, 43

Linsky, J. L., Wood, B. E., Brown, A., Giampapa, M. S., \& Ambruster, C. 1995, ApJ, 455, 670

Luck, R. E. 2017, AJ, 153, 21

Luck, R. E. 2018, AJ, 155, 111

Luger, R., Sestovic, M., Kruse, E., et al. 2017, Nat. Astron., 1, 0129

Luhman, K. L. 2014, ApJ, 786, L18

Marsden, S. C., Donati, J. F., Semel, M., Petit, P., \& Carter, B. D. 2006a, MNRAS, 370, 468

Marsden, S. C., Mengel, M. W., Donati, F., et al. 2006b, ASP Conf. Ser., 358, 401

Mauk, B. H., \& Bagenal, F. 2012, Geophys. Monograph Ser. 197, 3

Mauk, B. H., Haggerty, D. K., Paranicas, C., et al. 2017, Geophys. Res. Lett., 44, 4410

McComas, D. J., Alexashov, D., Bzowski, M., et al. 2012, Science, 336, 1291

Miliordos, E., \& Mavridis, A. 2010, J. Phys. Chem. A, 114, 8536

Morin, J., Donati, J. F., Forveille, T., et al. 2008a, MNRAS, 384, 77

Morin, J., Donati, J. F., Petit, P., et al. 2008b, MNRAS, 390, 567

Morin, J., Donati, J. F., Petit, P., et al. 2010, MNRAS, 407, 2269

Moutou, C., Donati, J. F., Savalle, R., et al. 2007, A\&A, 473, 651

Neiner, C., Geers, V. C., Henrichs, H. F., et al. 2003, A\&A, 406, 1019

Neubauer, F. M. 1980, J. Geophys. Res., 85, 1171

Neubauer, F. M. 1998, J. Geophys. Res., 103, 19843

Nichols, J. D., Burleigh, M. R., Casewell, S. L., et al. 2012, ApJ, 760, 59

Nieva, M.-F., \& Przybilla, N. 2014, A\&A, 566, A7

North, P. 1998, A\&A, 334, 181

Palmer, H. B., \& Hsu, C. J. 1972, J. Mol. Spectr., 43, 320

Pasinetti Fracassini, L. E., Pastori, L., Covino, S., \& Pozzi, A. 2001, A\&A, 367, 521

Pavlenko, Y. V., Jones, H. R. A., Lyubchik, Y., Tennyson, J., \& Pinfield, D. J. 2006, A\&A, 447, 709

Payne, M. J., \& Lodato, G. 2007, MNRAS, 381, 1597

Petit, P., Dintrans, B., Solanki, S. K., et al. 2008, MNRAS, 388, 80

Phan-Bao, N., Lim, J., Donati, J.-F., Johns-Krull, C. M., \& Martín, E. L. 2009, ApJ, 704, 1721

Phatak, C. M., \& Palmer, H. B. 1970, J. Mol. Spectr., 33, 137

Pineda, J. S., Hallinan, G., \& Kao, M. M. 2017, ApJ, 846, 75

Prangé, R., Rego, D., Southwood, D., et al. 1996, Nature, 379, 323

Pryor, W. R., Ajello, J. M., Tobiska, W. K., et al. 1998, J. Geophys. Res., 103, 20149 
Rabus, M., Lachaume, R., Jordán, A., et al. 2019, MNRAS, 484, 2674

Redfield, S., \& Linsky, J. L. 2008, ApJ, 673, 283

Rees, M. H. 1989, Physics and Chemistry of the Upper Atmosphere (New York: Cambridge University Press)

Reid, I. N. 2013, in Planets, Stars and Stellar Systems, Stellar Structure and Evolution, eds. T. D. Oswalt, \& M. A. Barstow (Berlin: Springer), 4, 337

Reid, I. N., Cruz, K. L., Laurie, S. P., et al. 2003, AJ, 125, 354

Reiners, A. 2012, Liv. Rev. Sol. Phys., 9, 1

Reiners, A., \& Basri, G. 2007, ApJ, 656, 1121

Reiners, A., \& Basri, G. 2008, A\&A, 489, L45

Reiners, A., \& Basri, G. 2009, ApJ, 705, 1416

Reiners, A., \& Basri, G. 2010, ApJ, 710, 924

Reiners, A., Zechmeister, M., Caballero, J. A., et al. 2018, A\&A, 612, A49

Riley, A., Debes, J., \& Lockwood, S. 2017, Instrument Science Report STIS 2017-5

Rodríguez-Barrera, M. I., Helling, C., \& Wood, K. 2018, A\&A, 618, A107

Rojas-Ayala, B., Covey, K. R., Muirhead, P. S., \& Lloyd, J. P. 2012, ApJ, 748, 93

Saur, J. 2004, J. Geophys. Res., 109, A01210

Saur, J., Grambusch, T., Duling, S., Neubauer, F. M., \& Simon, S. 2013, A\&A, 552, A119

Saur, J., Duling, S., Roth, L., et al. 2015, J. Geophys. Res. Space Phys., 120, 1715

Saur, J., Fischer, C., Wennmacher, A., et al. 2018a, ApJ, 859, 74

Saur, J., Janser, S., Schreiner, A., et al. 2018b, J. Geophys. Res. Space Phys., 123 9560

Schmidt, S. J., Hawley, S. L., West, A. A., et al. 2015, AJ, 149, 158

Ségransan, D., Kervella, P., Forveille, T., \& Queloz, D. 2003, A\&A, 397, L5

Seidelmann, P. K., Abalakin, V. K., Bursa, M., et al. 2002, Celest. Mech. Dyn. Astron., 82, 83

Shkolnik, E., Walker, G. A. H., Bohlender, D. A., Gu, P., \& Kürster, M. 2005 ApJ, 622, 1075

Shkolnik, E., Bohlender, D. A., Walker, G. A. H., \& Collier Cameron, A. 2008, ApJ, 676, 628

Showman, A. P., \& Malhotra, R. 1999, Science, 296, 77

Smith, R. A., \& Strobel, D. F. 1985, J. Geophys. Res., 90, 9469
Snow, T. P., Lamers, H. J. G. L. M., Lindholm, D. M., \& Odell, A. P. 1994, ApJS, 95, 163

Strobel, D. F., \& Atreya, S. K. 1983, in Physics of the Jovian Magnetosphere, ed. A. J. Dessler (Cambridge: Cambridge University Press), 51

Strugarek, A., Brun, A. S., Matt, S. P., \& Réville, V. 2015, ApJ, 815, 111

Takeda, G., Ford, E. B., Sills, A., et al. 2007, ApJS, 168, 297

Tannock, M. E., Metchev, S., Heinze, A., et al. 2021, AJ, 161, 224

Thébault, E., Finlay, C. C., Beggan, C. D., et al. 2015, Earth Planets Space, 67, 79

Turner, J. D., Zarka, P., Grießmeier, J.-M., et al. 2021, A\&A, 645, A59

Turnpenney, S., Nichols, J. D., Wynn, G. A., \& Casewell, S. L. 2017, MNRAS, 470,4274

Turnpenney, S., Nichols, J. D., Wynn, G. A., \& Burleigh, M. R. 2018, ApJ, 854, 72

van Belle, G. T., \& von Braun, K. 2009, ApJ, 694, 1085

Van Grootel, V., Fernandes, C. S., Gillon, M., et al. 2018, ApJ, 853, 30

Vedantham, H. K., Callingham, J. R., Shimwell, T. W., et al. 2020a, Nat. Astron., 4, 577

Vedantham, H. K., Callingham, J. R., Shimwell, T. W., et al. 2020b, ApJ, 903 , L33

Vrba, F. J., Henden, A. A., Luginbuhl, C. B., et al. 2004, AJ, 127, 2948

Wade, G. A., Neiner, C., Alecian, E., et al. 2016, MNRAS, 456, 2

Wannawichian, S., Clarke, J. T., \& Nichols, J. D. 2010, J. Geophys. Res. Space Phys., 115, A02206

Wolven, B. C., \& Feldman, P. D. 1998, Geophys. Res. Lett., 25, 1537

Woods, T. N., Eparvier, F. G., Bailey, S. M., et al. 2005, J. Geophys. Res. Space Phys., 110, 1312

Wright, J. T., Marcy, G. W., Butler, R. P., \& Vogt, S. S. 2004, ApJS, 152, 261

Yadav, R. K., Christensen, U. R., Morin, J., et al. 2015, ApJ, 813, L31

Youngblood, A., France, K., Loyd, R. O. P., et al. 2016, ApJ, 824, 101

Zarka, P. 1998, J. Geophys. Res., 103, 20159

Zarka, P. 2007, Planet. Space Sci., 55, 598

Zarka, P., Treumann, R. A., Ryabov, B. P., \& Ryabov, V. B. 2001, Astrophys. Space Sci., 277, 293

Zorec, J., \& Royer, F. 2012, A\&A, 537, A120 


\section{Appendix A: References and details of objects for auroral power potential calculations}

Table A.1 lists the objects for which magnetic fields, rotation rates, and auroral power potential are discussed in Section 2.3. The references used in Table A.1 are as follows:

(1): Showman \& Malhotra (1999), (2): Seidelmann et al. (2002), (3): Delitsky \& Lane (1998), (4): Kivelson et al. (2002) (5): NASA/JPL, retrieved from https://ssd.jpl.nasa.gov/?planet_phys_par, (6): NASA/JPL, retrieved from https://solarsystem. nasa.gov/resources/681/solar-system-temperatures/,

(7): Anderson et al. (2011), (8): Thébault et al. (2015), (9): Connerney et al. (1993), (10): Dougherty et al. (2018), (11): Connerney et al. (2018), (12): Turner et al. (2021), (13): Brogi et al. (2012), (14): Kao et al. (2016), (15): Kao et al. (2018), (16): Berdyugina et al. (2017), (17): Hallinan et al. (2008), (18): Morin et al. (2010), (19): Linsky et al. (1995), (20): Reiners \& Basri (2007), (21): Van Grootel et al. (2018), (22): Luger et al. (2017), (23): Delrez et al. (2018), (24): Casagrande et al. (2008), (25): Gaia Collaboration . (2018), (26): Pavlenko et al. (2006), (27): Reiners \& Basri (2010), (28): Ségransan et al. (2003), (29): Reiners \& Basri (2008), (30): Ségransan et al. (2003), (31): Lépine et al. (2013), (32): Reiners \& Basri (2009), (33): Morin et al. (2008b), (34): Morin et al. (2008a), (35): Takeda et al. (2007), (36): Boro Saikia et al. (2016), (37): Bouchy et al. (2005), (38): Henry \& Winn (2008), (39): Moutou et al. (2007), (40): Kervella et al. (2016). (41): Barnes et al. (2016),(42): Phan-Bao et al. (2009), (43): Gaidos et al. (2014), (44): Rabus et al. (2019), (45): Reiners et al. (2018), (46): Gaidos \& Mann (2014), (47): Rojas-Ayala et al. (2012), (48): van Belle \& von Braun (2009), (49): Kovári et al. (2004), (50): Donati (1999), (51): Luck (2018), (52): Luck (2017), (53): Boyajian et al. (2015), (54): Baines \& Armstrong (2012) (55): Fröhlich (2007), (56): Clem et al. (2004), (57): Basri \& Marcy (1988), (58): Petit et al. (2008), (59): Gaulme et al. (2010) (60): Grieves et al. (2018), (61): IAU 2015 resolution B3, (62): Jones (2004), (63): Banaszkiewicz et al. (1998), (64): Marsden et al. (2006a), (65): Marsden et al. (2006b), (66): Fossati et al. (2013), (67): Bonfanti et al. (2016), (68): Wright et al. (2004), (69): Kim \& Demarque (1996), (70): Blackwell \& Lynas-Gray (1994), (71): Borsa et al. (2015), (72): Donati et al. (2008), (73): North (1998), (74): Wade et al. (2016), (75): Zorec \& Royer (2012), (76): Hubrig et al. (2009), (77): Neiner et al. (2003), (78): Snow et al. (1994), (79): Pasinetti Fracassini et al. (2001), (80): Hohle et al. (2010), (81): Nieva \& Przybilla (2014),

The B-field values from planets, brown dwarfs, and stars are obtained with different measurement techniques. Details on the remote sensing methods can be found in Reiners (2012), among others. The magnetic field measurements from Table A.1 are based on the following methods: 1: In situ, 2: Radio ECM, 3: Bf (average magnetic flux from Stokes I), 4: $\langle\mathrm{Bz}\rangle$ (average measured longitudinal field strength from Stokes V), 5: $B z_{\max }$ (maximum measured longitudinal field strength from Stokes V), 6: $\langle\mathrm{B}\rangle$ (average field strength from ZDI map with Stokes V and I), 7: $B_{\max }$ (maximum field strength from ZDI map with Stokes $\mathrm{V}$ and I), d: $B_{\max }$ (maximum field strength from ZDI map with Stokes $\mathrm{V}$ only), e: $B z_{\max }$ (maximum measured longitudinal field strength from Stokes V, Q, and U).

\section{Appendix B: Further details of observations}

\section{Appendix B.1: Data and error analysis}

For the analysis of the observations we primarily worked with the highest calibration level of STIS data provided within the $x 2 d$-files where spectral energy fluxes in erg s $\mathrm{s}^{-1} \mathrm{~cm}^{-2} \AA^{-1}$ are given. Because the target is extremely faint in the UV, the position of the target within the slit could not be directly determined. We therefore used the reference position provided in the headers

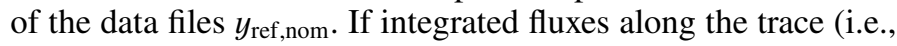
the direction of dispersion) in individual or combined exposures show locally enhanced fluxes near the expected location of the target, we worked with the improved position. We neglected pixels on the detector where data quality flags indicate quality issues for a given exposure and subsequently renormalized integrated fluxes considering the missing pixels.

Similar to the analysis in Saur et al. (2018a), the spectral flux as a function of wavelength $\lambda$ represented by column $i_{x}$ is given by

$f_{\text {trace }}(\lambda)=\sum_{i_{y}=y_{\text {ref }}-n_{1}}^{y_{\text {ref }}+n_{2}} f\left(\lambda, i_{y}\right)$.

We calculate the average background flux per pixel $f_{b g}^{p x}$ in rows sufficiently above and below the rows where we expect flux from the target with

$f_{b g}^{p x}(\lambda)=\frac{\sum_{i_{y}=y_{r e f}+a_{1}}^{y_{r e f}+a_{2}} f\left(\lambda, i_{y}\right)+\sum_{i_{y}=y_{r e f}-b_{1}}^{y_{r e f}-b_{2}} f\left(\lambda, i_{y}\right)}{a_{2}-a_{1}+1+b_{2}-b_{1}+1}$,

with the positive integer numbers $n_{1}, n_{2}, a_{1}, a_{2}, b_{1}, b_{2}$. The values of these integers depend on the type of aperture and grating for each exposure (see Table B.1). In these calculations a separate background is calculated for each column $i_{x}$ because the background fluxes change along the dispersion axis. The net flux from the target as a function of wavelength is thus given by

$f_{\text {net }}(\lambda)=f_{\text {trace }}(\lambda)-f_{\text {bg }}^{p x}(\lambda)\left(n_{2}-n_{1}+1\right)$.

We calculate the variances and the $\mathrm{S} / \mathrm{N}$ in two ways. The variances of the background for a certain wavelength $\lambda$ represented by an individual column $i_{x}$ is given by

$$
\begin{aligned}
V^{p x}\left(i_{x}\right)= & \left(\sum_{i_{y}=y_{\text {ref }}+a_{1}}^{y_{\text {ref }}+a_{2}}\left(f\left(i_{x}, i_{y}\right)-f_{b g}^{p x}\left(i_{x}\right)\right)^{2}\right. \\
& \left.+\sum_{y_{\text {ref }}-b_{1}}^{y_{\text {ref }}-b_{2}}\left(f\left(i_{x}, i_{y}\right)-f_{b g}^{p x}\left(i_{x}\right)\right)^{2}\right) \\
& /\left(a_{2}-a_{1}+b_{2}-b_{1}+1\right) .
\end{aligned}
$$

For comparison of net fluxes $f_{\text {net }}$ from the target with flux uncertainties, the variance in each pixel $V^{p x}$ needs to be normalized to the number of rows used to calculate $f_{\text {net }}$. For the calculation of net fluxes within certain wavelength ranges, the variances of the individual columns within the selected wavelength ranges need to be summarized to the total variance $V$. The $\mathrm{S} / \mathrm{N}$ values are finally calculated with the standard deviation $\sigma=\sqrt{V}$.

Alternatively, we calculate the $\mathrm{S} / \mathrm{N}$ based on the counts that contribute to the calculation of certain fluxes (based on $x 2 d$-files or $f t$-files) using

$S / N=\frac{S-B}{\sqrt{S+B}}$, 


\begin{tabular}{|c|c|c|c|c|c|c|c|}
\hline Object name & Classification & $\mathbf{M}[\mathrm{kg}]$ & $\mathrm{R}[\mathrm{m}]$ & $\mathrm{P}[\mathrm{h}]$ & $T_{e f f}[\mathrm{~K}]$ & $\mathrm{B}[\mathrm{G}]$ & B-meth ${ }^{f}$ \\
\hline Ganymede & Moon & $1.43 \times 10^{23}(1)$ & $2.63 \times 10^{6}(2)$ & $171.71(2)$ & $110(3)$ & $0.0144(4)$ & 1 \\
\hline Mercury & Planet & $3.30 \times 10^{23}(5)$ & $2.44 \times 10^{6}(2)$ & $1407.51(2)$ & $703(6)$ & $0.004(7)$ & 1 \\
\hline Earth & Planet & $5.972 \times 10^{24}(5)$ & $6.37 \times 10^{6}(2)$ & $23.93(2)$ & $289(6)$ & $0.6(8)$ & 1 \\
\hline Uranus & Planet & $8.68 \times 10^{25}(5)$ & $2.54 \times 10^{7}(2)$ & $17.24(2)$ & $78(6)$ & $1.0(9)$ & 1 \\
\hline Neptune & Planet & $1.02 \times 10^{26}(5)$ & $2.46 \times 10^{7}(2)$ & $16.11(2)$ & $72(6)$ & $0.9(9)$ & 1 \\
\hline Saturn & Planet & $5.683 \times 10^{26}(5)$ & $5.82 \times 10^{7}(2)$ & $10.66(2)$ & $135(6)$ & $0.42(10)$ & 1 \\
\hline Jupiter & Planet & $1.898 \times 10^{27}(5)$ & $6.991 \times 10^{7}(2)$ & $9.92(2)$ & $165(6)$ & $20(11)$ & 1 \\
\hline$\tau$ Boo b & Exoplanet & $1.129 \times 10^{28}(13)$ & $8.0 \times 10^{7}(12)$ & $79.49(13)$ & $1650(13)$ & $10.7(12)$ & 2 \\
\hline 2MASS J10430758+2225236 & BD L8 & $2.187 \times 10^{28}(14)$ & $6.292 \times 10^{7}(\mathrm{a})$ & $2.21(15)$ & $1012(14)$ & $3900(15)$ & 2 \\
\hline SDSS J04234858-0414035 & $\mathrm{BD} \mathrm{L} 7+\mathrm{T} 2.5$ & $2.983 \times 10^{28}(14)$ & $6.292 \times 10^{7}(\mathrm{a})$ & $1.47(15)$ & $1084(14)$ & $3900(15)$ & 2 \\
\hline SIMP J01365662+0933473 & BD T2.5 & $4.375 \times 10^{28}(14)$ & $6.292 \times 10^{7}(\mathrm{a})$ & $2.88(15)$ & 1104 (14) & $3200(15)$ & 2 \\
\hline 2MASS J10475385+2124234 & BD T6.5 & $>5.170 \times 10^{28}(14)$ & $6.292 \times 10^{7}(\mathrm{a})$ & $1.78(15)$ & $888(14)$ & $5600(15)$ & 2 \\
\hline 2MASS J12373919+6526148 & BD T6.5 & $>5.568 \times 10^{28}(14)$ & $6.292 \times 10^{7}(\mathrm{a})$ & $2.28(15)$ & $851(14)$ & $4100(15)$ & 2 \\
\hline 2MASS J18353790+3259545 & * M8.5 & $1.044 \times 10^{29}(16)$ & $1.468 \times 10^{8}(16)$ & $2.84(17)$ & $2800(16)$ & $5100(16)$ & 2 \\
\hline VB 10 & * M8 & $1.591 \times 10^{29}(18)$ & $6.26 \times 10^{7}(18)$ & $19.2\left(68^{c}\right)$ & $2600(19)$ & $1300(20)$ & 3 \\
\hline Trappist-1 & * M8 & $1.770 \times 10^{29}(21)$ & $8.42 \times 10^{7}(21)$ & $79.2(22)$ & $2511(23)$ & $600(27)$ & 3 \\
\hline GJ 3622 & * M6.5 & $1.790 \times 10^{29}(18)$ & $7.65 \times 10^{7}(18)$ & $36(18)$ & $2450(24)$ & $110(18)$ & 7 \\
\hline VB 8 & $* \mathrm{M} 7$ & $1.790 \times 10^{29}(18)$ & $6.96 \times 10^{7}(18)$ & $24\left(16^{c}\right)$ & $3299(25)$ & $2300(20)$ & 3 \\
\hline WX Uma & * M6 & $1.988 \times 10^{29}(18)$ & $8.35 \times 10^{7}(18)$ & $18.72(18)$ & $2700(24)$ & $4880(18)$ & 7 \\
\hline DX Cnc & * M6 & $1.988 \times 10^{29}(18)$ & $7.65 \times 10^{7}(18)$ & $11.04(18)$ & $2840(20)$ & $220(18)$ & 7 \\
\hline CN Leo & * M5.5 & $1.988 \times 10^{29}(18)$ & $8.35 \times 10^{7}(18)$ & $48\left(16^{c}\right)$ & $2800(26)$ & $2400(20)$ & 3 \\
\hline GJ 1245 B & * M5.5 & $2.386 \times 10^{29}(18)$ & $9.74 \times 10^{7}(18)$ & $17.04(18)$ & $3294(25)$ & $580(18)$ & 7 \\
\hline Proxima Centauri & * M5.5 & $2.446 \times 10^{29}(28)$ & $1.01 \times 10^{8}(28)$ & $1992(29)$ & $3042(30)$ & $600(29)$ & 3 \\
\hline GJ 1156 & $*$ M5 & $2.784 \times 10^{29}(18)$ & $1.11 \times 10^{8}(18)$ & $11.78(18)$ & $3110(31)$ & $360(18)$ & 7 \\
\hline GJ 1224 & * M4.5 & $2.983 \times 10^{29}(18)$ & $1.18 \times 10^{8}(18)$ & $103.2\left(16^{c}\right)$ & $3300(10)$ & $2700(20)$ & 3 \\
\hline GJ $1154 \mathrm{~A}$ & * M5 & $3.580 \times 10^{29}(18)$ & $1.39 \times 10^{8}(18)$ & $40.8\left(16^{c}\right)$ & $3040(31)$ & $2000(32)$ & 3 \\
\hline GJ 51 & * M5 & $4.375 \times 10^{29}(18)$ & $1.53 \times 10^{8}(18)$ & $24.48(18)$ & $3346(25)$ & $5020(18)$ & 7 \\
\hline V374 Peg & $* \mathrm{M} 4$ & $5.568 \times 10^{29}(33)$ & $1.948 \times 10^{8}(33)$ & $10.7(33)$ & $3432(25)$ & $1300(34)$ & 7 \\
\hline GJ $65 \mathrm{~B}$ & * M6 & $2.376 \times 10^{29}(40)$ & $1.11 \times 10^{8}(40)$ & $5.52(41)$ & $3296(25)$ & $5800(41)$ & 3 \\
\hline GJ $65 \mathrm{~A}$ & * M5.5 & $2.436 \times 10^{29}(40)$ & $1.15 \times 10^{8}(40)$ & $5.76(41)$ & $3337(25)$ & $4500(41)$ & 3 \\
\hline Gl 490 B & * M4 V & $2.983 \times 10^{29}(42)$ & $3.27 \times 10^{8}(42)$ & $12.1(42)$ & $3266(43)$ & $1800(42)$ & 7 \\
\hline Gl 729 & $* \mathrm{M} 3.5$ & $3.480 \times 10^{29}(44)$ & $1.426 \times 10^{8}(44)$ & $68.88(45)$ & $3162(44)$ & $2200(20)$ & 3 \\
\hline EQ Peg B & $* \mathrm{M} 4.5$ & $4.971 \times 10^{29}(33)$ & $1.739 \times 10^{8}(33)$ & $9.70(33)$ & $3309(25)$ & $1200(33)$ & 7 \\
\hline YZ CMi & * M4.5 & $6.164 \times 10^{29}(33)$ & $2.017 \times 10^{8}(33)$ & $66.62(33)$ & $3125(46)$ & $3000(33)$ & 7 \\
\hline EV Lac & * M3.5 & $6.363 \times 10^{29}(33)$ & $2.087 \times 10^{8}(33)$ & $104.92(33)$ & 3400 (47) & $1500(33)$ & 7 \\
\hline EQ Peg A & * M3.5 & $7.755 \times 10^{29}(33)$ & $2.435 \times 10^{8}(33)$ & $25.46(33)$ & 3585 (25) & $800(33)$ & 7 \\
\hline AD Leo & $* \mathrm{M} 3$ & $8.352 \times 10^{29}(33)$ & $2.643 \times 10^{8}(33)$ & $53.76(33)$ & $3390(47)$ & $1300(33)$ & 7 \\
\hline 61 Cyg A & $* \mathrm{~K} 5 \mathrm{~V}$ & $1.321 \times 10^{30}(35)$ & $4.31 \times 10^{8}(35)$ & $856.8(36)$ & $4526(48)$ & $20(36)$ & 7 \\
\hline LQ Hya & $* \mathrm{~K} 0 \mathrm{~V}$ & $1.591 \times 10^{30}(49)$ & $5.57 \times 10^{8}(50)$ & $38.4(50)$ & $4812(51)$ & $800(50)$ & 7 \\
\hline HD 189733 & $* \mathrm{~K} 2$ & $1.631 \times 10^{30}(37)$ & $5.29 \times 10^{8}(37)$ & $286.87(38)$ & 4875 (53) & $40(39)$ & 7 \\
\hline$\epsilon$ Eri & $* \mathrm{~K} 2 \mathrm{~V}$ & $1.631 \times 10^{30}(54)$ & $5.009 \times 10^{8}(55)$ & $268.80(55)$ & $5156(56)$ & $350(57)$ & 3 \\
\hline HR 1099 & $* \mathrm{~K} 1 \mathrm{IV}$ & $1.790 \times 10^{30}(50)$ & $2.3 \times 10^{9}(50)$ & $67.2(50)$ & $3266(52)$ & $800(50)$ & 7 \\
\hline HD 190771 & * G2 V C & $1.909 \times 10^{30}(58)$ & $6.957 \times 10^{8}(58)$ & $211.2(58)$ & $5834(58)$ & $51(58)$ & 6 \\
\hline HD 46375 & $* \mathrm{~K} 0 \mathrm{~V}$ & $1.929 \times 10^{30}(59)$ & $6.33 \times 10^{8}(59)$ & 1008 (59) & $5355(60)$ & $5(59)$ & $7^{d}$ \\
\hline $18 \mathrm{Sco}$ & * G2 Va B & $1.949 \times 10^{30}(58)$ & $6.957 \times 10^{8}(58)$ & $544.8(58)$ & $5791(58)$ & $3.6(58)$ & 6 \\
\hline Sun & * G2V & $1.988 \times 10^{30}(61)$ & $6.957 \times 10^{8}(1)$ & $609.12(2)$ & $5780(62)$ & $10(63)$ & 1 \\
\hline V* V401 Нуа & * G8/K0(IV) E & $2.008 \times 10^{30}(58)$ & $6.957 \times 10^{8}(58)$ & $295.2(58)$ & $5802(58)$ & $42(58)$ & 6 \\
\hline HD 171488 & $* \mathrm{G} 2 \mathrm{~V}$ & $2.108 \times 10^{30}(64)$ & $8.00 \times 10^{30}(64)$ & $31.2(65)$ & $5800(64)$ & $500(65)$ & 7 \\
\hline HD 117207 & * G7 IV-V C & $2.148 \times 10^{30}(66)$ & $7.861 \times 10^{8}(67)$ & $864(68)$ & $5681(67)$ & $1.36(66)$ & 4 \\
\hline HD 1817 & $* \mathrm{~F} 8 \mathrm{~V}$ & $2.287 \times 10^{30}(69)$ & $8.21 \times 10^{8}(70)$ & $24(65)$ & $6126(51)$ & $250(65)$ & 7 \\
\hline HD 76151 & * G2 V B & $2.466 \times 10^{30}(58)$ & $6.957 \times 10^{8}(58)$ & $492(58)$ & $5790(58)$ & $5.6(58)$ & 6 \\
\hline$\tau$ Boo A & * F7 IV & $2.76 \times 10^{30}(71)$ & $9.878 \times 10^{8}(71)$ & $79.44(72)$ & $6399(71)$ & $10(72)$ & 7 \\
\hline a Cen & * B7 IIIpv & $7.357 \times 10^{30}(73)$ & $3.958 \times 10^{9}(73)$ & $211.68(74)$ & $17700(73)$ & $470(74)$ & 5 \\
\hline$\epsilon$ Dor & $* \mathrm{~B} 6 \mathrm{~V}$ & $8.57 \times 10^{30}(75)$ & $2.643 \times 10^{9}(76)$ & $>271.00(75)$ & $13700(76)$ & $64(76)$ & 4 \\
\hline$\zeta$ Cas & $* \mathrm{~B} 2 \mathrm{IV}$ & $1.65 \times 10^{31}(77)$ & $4.104 \times 10^{9}(77)$ & $128.88(74)$ & $20426(77)$ & $30(74)$ & 5 \\
\hline V1046 Ori & $* \mathrm{~B} 1.5 \mathrm{~V}$ & $1.988 \times 10^{31}\left(41^{b}\right)$ & $3.130 \times 10^{9}(79)$ & $21.6(74)$ & $23700(80)$ & $2035(74)$ & 5 \\
\hline V901 Ori & $* \mathrm{~B} 2 \mathrm{~V}$ & $1.988 \times 10^{31}(78)$ & $4.591 \times 10^{9}(78)$ & $36.96(74)$ & $22000(78)$ & $1310(74)$ & $5^{e}$ \\
\hline HD 64740 & $* \mathrm{~B} 1.5 \mathrm{Vp}$ & $1.988 \times 10^{31}\left(41^{b}\right)$ & $5.843 \times 10^{9}(79)$ & $31.92(74)$ & $23700(80)$ & $660(74)$ & 5 \\
\hline$\beta$ Cep & * B1 IV & $2.426 \times 10^{31}(81)$ & $3.896 \times 10^{9}(81)$ & $288(74)$ & $27000(81)$ & $110(74)$ & 5 \\
\hline NU Ori & $* \mathrm{~B} 4$ & $2.983 \times 10^{31}(78)$ & $5.426 \times 10^{9}(78)$ & $15.12(74)$ & $27700(78)$ & $310(74)$ & 5 \\
\hline$\tau \mathrm{Sco}$ & $* \mathrm{~B} 0 \mathrm{~V}$ & $2.983 \times 10^{31}(78)$ & $4.174 \times 10^{9}(78)$ & $984.72(74)$ & $30000(78)$ & $90(74)$ & 5 \\
\hline
\end{tabular}

Table A.1. Mass $M$, radius $R$, period $P$, effective temperature $T_{e f f}$, and surface magnetic field $B$ for the objects in this study. The sources for these values are indicated in parentheses with the associated references given in the main text of the Appendix. Footnotes: a: Typical brown dwarf radius of $0.9 R_{\text {Jup }}$ assumed (Vrba et al. 2004; Kao et al. 2016); b: Mass derived from spectral type using the table in the indicated reference; c: Maximum rotational period estimated in indicated reference; $\mathrm{f}$ : Magnetic field values are based on different observational methods listed in the last column with the individual technique given in the main text of the Appendix. 
Table B.1. Details of the data analysis. Row information for calculation of fluxes and $\mathrm{S} / \mathrm{N}$.

\begin{tabular}{cccccccc}
\hline \hline Type & $y_{r}$ & $n_{1}$ & $n_{2}$ & $a_{1}$ & $a_{2}$ & $b_{1}$ & $b_{2}$ \\
\hline NUV & 604 & -5 & 5 & 6 & 26 & -26 & -6 \\
FUV & 476 & -5 & 5 & 6 & 46 & -26 & -6 \\
Ly- $\alpha$ & 486 & -5 & 5 & 6 & 36 & -36 & -6 \\
\hline
\end{tabular}

with the counts of the total signal $S$ and the background $B$. The signal and the background are taken from the same pixels as described in Sects. (B.3) and (B.4) and Table B.1.

\section{Appendix B.2: Acquisition of target}

Figure B.1 shows an image of the first acquisition exposure of visit 1 blind-pointing into an area of 5 by 5 arcsec. The brightest object in Figure B.1 is located at RA $=12: 37: 35.7807$ and $\mathrm{DEC}=65: 26: 03.250$. The standard deviations for the expected positional accuracy in right ascension and declination of the target in the acquisition image has been determined as the root sum squared of the standard deviations of the initial position of 2MASS J1237+6526 in the 2MASS catalogue (0.19", $0.18 ")$, of the proper motion correction (0.17", 0.12") (Vrba et al. 2004), and of the pointing accuracy of HST using stars in the guide star catalogue $\left(0.2^{\prime \prime}, 0.2^{\prime \prime}\right)$ as $\left(0.32^{\prime \prime}, 0.30^{\prime \prime}\right)$. The offset of the brightest object from the expected position is $(-0.07 ", 0.47 ")$, hence we derive a total positional offset of $1.6 \sigma$. The brightness of the object in the acquisition image corresponds to $43 \%$ of the expected brightness of the target. This estimate stems from runs with the STIS exposure time calculator using the spectrum of 2MASS J1237+6526 from Burgasser et al. (2002) and Liebert \& Burgasser (2007).

Separated by 2.6 ", there is a second slightly dimmer object in the acquisition image. Its location is $7.0 \sigma$ away from the expected location of 2MASS J1237+6526 and its total counts in the acquisition exposure amount to $34 \%$ of the counts expected from 2 MASS $\mathbf{J} 1237+6526$. In particular due to the significant deviation from the expected position, we do not expect the second object to be our desired target. Unfortunately, we could not identify the second object within standard data catalogues.

The subsequent acquisition procedure by HST positioned the brightest object of the initial acquisition exposure into the desired slits of the science exposures. The acquisition image of visit 2 is basically identical.

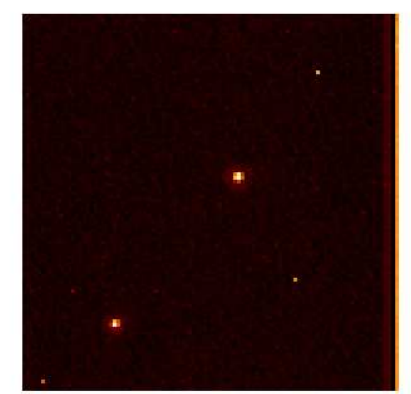

Fig. B.1. Acquisition image 